\title{
Performance of mixed formulations for the Particle Finite Element Method in soil mechanics problems
}

\author{
Lluís Monforte \\ Josep Maria Carbonell \\ Marcos Arroyo \\ Antonio Gens
}

\begin{abstract}
This paper presents a computational framework for the numerical analysis of fluid-saturated porous media at large strains. The proposal relies, on one hand, on the Particle Finite Element Method (PFEM), known for its capability to tackle large deformations and rapid changing boundaries, and, on the other hand, on constitutive descriptions well-established in current geotechnical analyses (Darcy's law; Modified Cam Clay; Houlsby hyper-elasticity). An important feature of this kind of problem is that incompressibility may arise either from undrained conditions or as a consequence of material behavior; incompressibility may lead to volumetric locking of the low-order elements that are typically used in PFEM. In this work, two different three-field mixed formulations for the coupled hydro-mechanical problem are presented, in which either the effective pressure or the Jacobian are considered as nodal variables, in addition to the solid skeleton displacement and water pressure. Additionally, several mixed formulations are described for the simplified single-phase problem due to its formal similitude to the poromechanical case and its relevance in geotechnics, since it may approximate the saturated soil behavior under undrained conditions. In order to use equal order interpolants in displacements and scalar fields, stabilization techniques are used in the mass conservation equation of the biphasic medium and in the rest of scalar equations. Finally, all mixed formulations are assessed in some benchmark problems and their performances are compared. It is found that mixed formulations that have the Jacobian as a nodal variable perform better.

Keywords: Particle Finite Element Method (PFEM), Finite Deformation, Mixed Formulations, Soil Mechanics.
\end{abstract}

\section{Introduction}

The Particle Finite Element Method (PFEM) is a numerical method well suited for mechanical problems involving large displacements, large deformations, intermittent separation and/or fusion of bodies. Soft porous materials such as soils suffer these kinds of mechanical transformations during many activities of engineering interest. Relevant examples for the case of soils include probing, sampling, pile installation, excavation and ploughing.

It is well known that PFEM originated to address problems of fluid mechanics [20], including those of fluid interaction with rigid bodies. Later it was extended to deformable single-phase solids [19]. Subsequently, several PFEM extensions have addressed geomaterials: Carbonell et al [10] used PFEM to simulate ground excavation problems. Flow-like landslides have been also studied using PFEM, but considering a singlephase material description. In Zhang et al [40] a rigid plastic constitutive response was assumed for the soil. Salazar et al [30] used a non-Newtonian modified Bingham law. Larese et al [15] presented a strategy to simulate the free surface flow over and throughout a rockfill.

For many porous materials, and particularly for water saturated soils, most observed mechanical responses cannot be explained without considering the fluid filling its pores. The appropriate general framework is that of poromechanics in which the continuum is considered to be composed of two phases (solid skeleton and water) whose interaction is expressed in appropriately formulated linear momentum and mass balance conservation laws. Extending PFEM to deal with poromechanics-based hydromechanical coupling seems then a necessary step to make the method relevant to a large class of soil mechanics problems. 
There are two extreme situations for hydromechanical coupling [24]. The first one corresponds to freely drained conditions. In this case, there is no change in the fluid pore pressure and changes in total and effective stress coincide. The second situation corresponds to fully undrained conditions: there is no relative motion between water and soil skeleton and the mixture behaves like an incompressible material. These extreme situations may be dealt with using simpler single-phase formulations. For instance, for undrained conditions, elasto-plastic formulations using a quasi-incompressible elastic model alongside an isochoric plastic law are applicable [17].

Simpler models have several advantages, amongst them faster computation, but their field of applicability is limited to purely drained or undrained situations. A fully coupled formulation is required to address the full range of potential geotechnical problems. Naturally, a fully coupled formulation should have both free draining and undrained behaviour as limiting cases.

Undrained conditions in water-saturated soils result in quasi-incompressible behavior. This causes a well-known numerical problem when using the Finite Element method: volumetric locking of low-order finite elements. Volumetric locking introduces numerical stiffening and spurious high spatial variability in the solution, eventually leading to numerical instability. The reason behind this behaviour is the failure to satisfy the Babuska-Brezzy conditions [2,8] or the equivalent inf-sup condition [3] due to an improper finitedimensional space in the finite element discretization. To avoid this problem two strategies are common: either to use more complex, but stable, finite elements [38, 26], or to apply stabilization procedures to originally unstable finite elements $[23,39,21]$. In the later approach, locking is mitigated at the cost of using a mixed formulation, thereby introducing extra degrees of freedom per node with respect to the primal formulation. Incompressibility may also arise under general drainage conditions in constitutive models of materials that predict zero volume change. This is the case, for instance, when failure is reached in Critical State soil models.

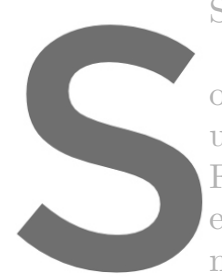

Addressing volumetric or in the single-phase un use of mixed formulatio First, in most cases it elements. Secondly, as may result from the mechanical b his
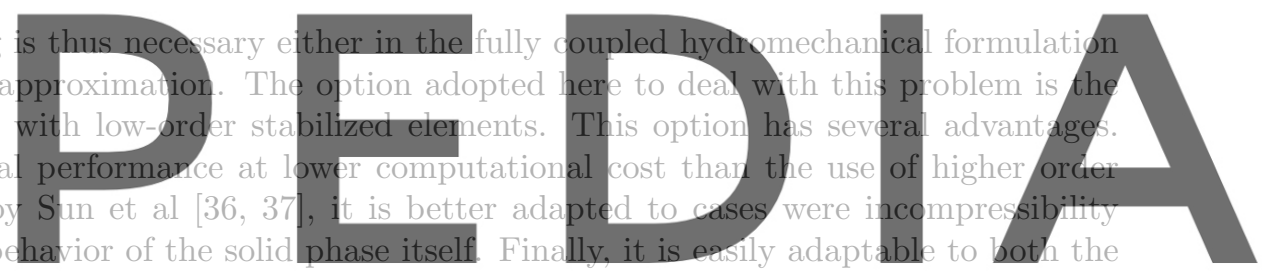

coupled hydromechanical and single-phase undrained formulations, taking advantage of their similar formal

In this work alternative mixed stabilized formulations both for total stress and for coupled hydromechanical analyses at large strains are explored. In the total stress case, only the linear momentum balance equation is solved with a nearly incompressible elastic model and an isochoric plastic law. In the coupled hydromechanical case, the system of equations of linear momentum and mass balance equations is solved using a monolithic approach whereas the solid skeleton follows a critical state plasticity model. Thus, volumetric locking caused either by incompressibility at nearly undrained conditions or by incompressibility of the solid skeleton are both dealt with.

The paper is structured as follows: first, the basics of the Particle Finite element method, are presented. Then, the balance equations for the mechanical problem and the hydromechanical problem are described in its primal and mixed form including the stabilization terms. After briefly reviewing the constitutive relations, the comparative performance of the different formulations implemented is explored via benchmark problems: first for the single-phase mechanical problem, and then for the hydromechanical problem. Finally, a number of conclusions are drawn.

\section{Fundamentals of PFEM for soil mechanics}

In PFEM the continuum is modelled using an Updated Lagrangian formulation; that is, a Lagrangian description of the motion is used and all variables are referred to the last known configuration. A mesh discretization of the domain must be generated in order to solve the governing equations in the standard FEM fashion. 
Nodes in that mesh are treated as material particles whose motion is tracked during the solution.

The quality of the numerical solution depends on the discretization chosen. The original idea of the PFEM was to improve the mesh quality by performing a re-triangulation of the domain only when needed. Usually that is performed according to some criteria associated with element distortion. Mesh distortion is corrected and improved naturally with the Particle Finite Element Method (PFEM), because re-triangulation is based on Delaunay tessellations that maximize the minimum angle of all the triangles in the tessellation. Therefore, thin, stretched elements are avoided while still capturing large changes in the continuum domain without global remeshing and mesh to mesh interpolation. The process can be easily extended to 3D using tetrahedral elements.

Additionally, $h$-adaptive techniques are employed to obtain a better discretization of the domain. New particles are introduced in areas where large gradients in the flow variables are detected or where a high plastic dissipation is generated. These zones must be refined because the number of particles may become too low to obtain an accurate solution. On the contrary, due to high shear deformations, particles may locally concentrate in the same region of the domain. To overcome this difficulty particles that are closer than a characteristic distance are removed.

Conforming meshes are used to preserve mass in the remeshing process: the boundary of the domain remains unchanged so the volume of the discretization does not vary. The mass of the domain also depends on the density and thus on the transfer process of Gauss point variables. This, however, is relatively unimportant for soil applications, since highest mesh distortion is usually associated with shearing and the attainment of incompressible critical state conditions.

Although it is not strictly necessary (see [40]), low order finite elements are typically used in PFEM:

linear triangles in two-dimensional models and linear tetrahedron in three dimensions. Linear interpolated elements have several advantages due to their simplicity: particles usually define exclusively the mesh nodes and no extra interpolations cost is lower compared to required instead.

The interpolation of are inserted in the domai To avoid excessive smoothing of internal variables, in
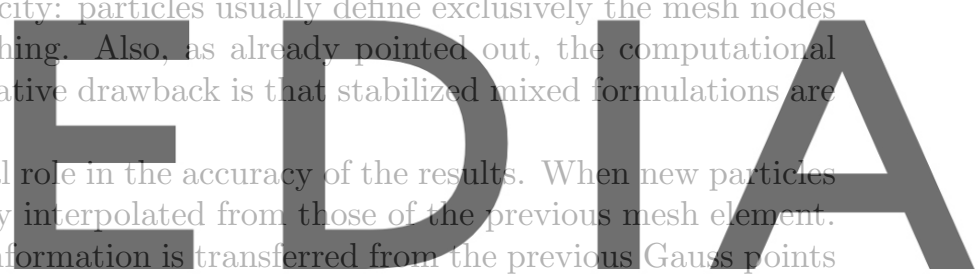

to the new ones. In this work, a nearest interpolation procedure is used; hence, new integration points inherit

strategies are possible, see for instance [25]. Extended details about remeshing and interpolation in PFEM applications for solid mechanics can be found in [28, 9].

A typical solution algorithm of PFEM is conceptually illustrated in Figure 1 for a general case of fragmentation and deformation of a solid mass under the action of an external object; (note, however, that fracture is not considered in this work). For clarity purposes we will define the collection or cloud of nodes (C) belonging to the analysis domain, the volume $(V)$ defining the analysis domain and the mesh $(M)$ discretizing the domain. The simulation involves the following steps:

1. Begin the computation of each time step with a cloud of points defining the analysis domain. For instance $C_{n}$ denotes the cloud at time $t=t_{n}$, see Figure 1.

2. Identify the boundaries defining the analysis domain $V_{n}$. This is an essential step as some boundaries may be severely distorted during the solution and, conceptually, may include separation and re-entering of nodes. The domain boundary may be identified with the $\alpha$-shape method [12] or that of the previous step, using conforming meshes.

3. Discretize the continuum domains with a finite element (FE) mesh $M_{n}$.

4. Solve the Lagrangian equations of motion in the domain. Compute the state variables at the next (updated) configuration for $t+\Delta t$ : displacements, pressure, water-pressure, strains, stresses and internal variables, etc. 


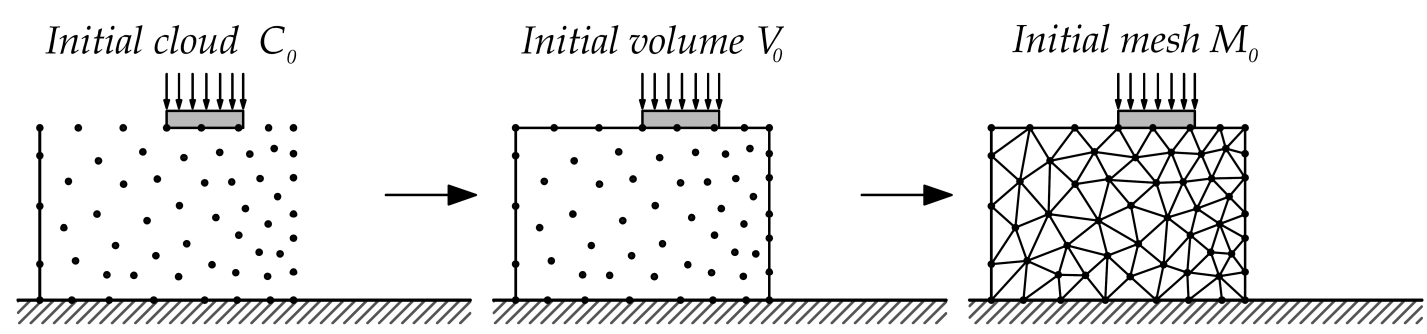

\section{Cloud at time $t_{n} C_{n} \quad$ Volume at time $t_{n} V_{n} \quad$ Mesh at time $t_{n} M_{n}$}
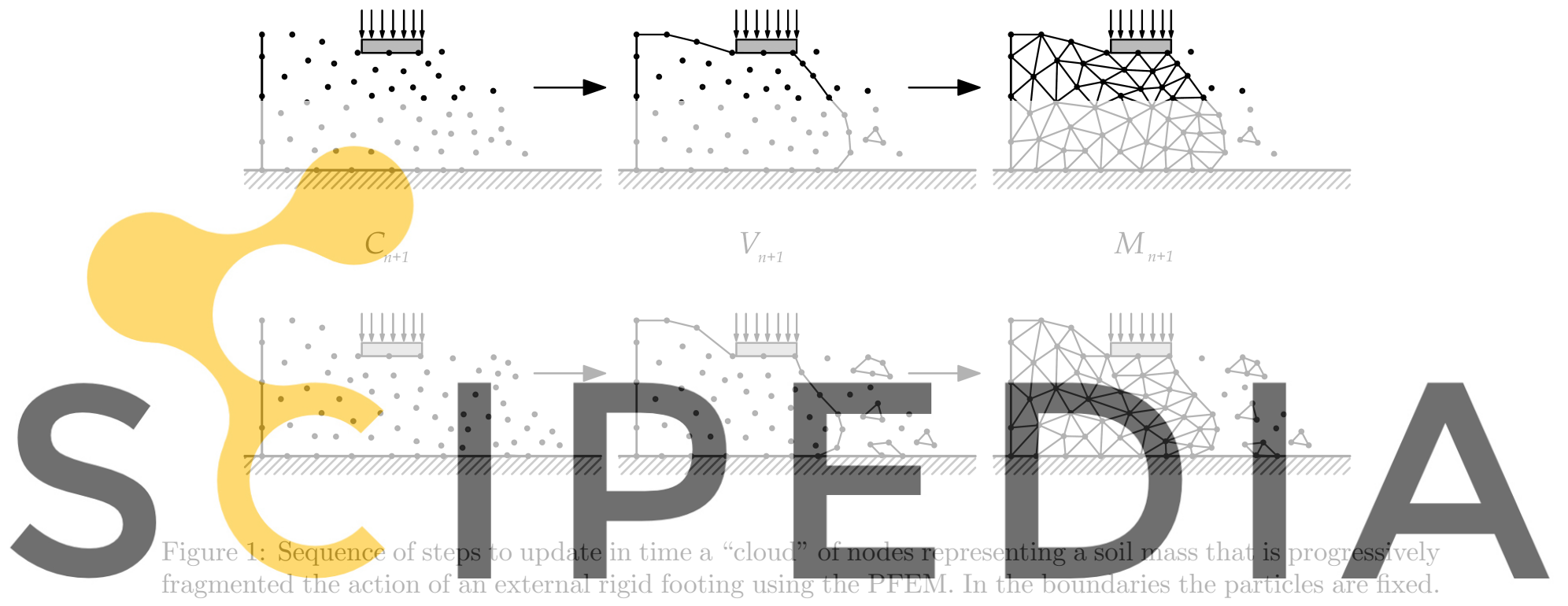

\section{Register for free at https//www.scipedia.com to download the version without the watermark 5. Move the mesh nodes to a new position $C_{n+1}$ where $n+1$ denotes the time $t_{n}+\Delta t$, in terms of the time increment size. This step is typically a consequence of the solution process of step 4.}

6. Go back to step 1 and repeat the solution process for the next time step to obtain $C_{n+2}$. The process is shown in Figure 1.

\section{$3 \quad$ Multiple field mixed formulations in finite strains}

This section presents the balance equations relevant for the two cases of single-phase continuous media and fluid-saturated porous media. The governing equations are presented both in their primal form and then using mixed forms. After describing the stabilization procedures, the stabilized Galerkin expressions of the formulations are presented.

\subsection{Single-phase mechanical media}

A quasi-static linear momentum displacement-based finite element formulation in updated Lagrangian form (i.e. expressing all quantities and their derivatives in the deformed configuration), valid for two- and threedimensional conditions may be written as: 


$$
\begin{cases}\nabla \cdot \boldsymbol{\sigma}+\mathbf{b}=\mathbf{0} & \text { in } \Omega_{t} \times(0, T) \\ \boldsymbol{u}(\boldsymbol{X}, t=0)=\boldsymbol{u}_{0} & \text { in } \Omega_{0} \\ \boldsymbol{u}(\boldsymbol{X}, t)=\overline{\boldsymbol{u}} & \text { in } \Gamma_{u} \times(0, T) \\ \boldsymbol{n} \cdot \boldsymbol{\sigma}=\overline{\boldsymbol{t}} & \text { in } \Gamma_{\bar{t}} \times(0, T)\end{cases}
$$

where $\boldsymbol{\sigma}=\hat{\boldsymbol{\sigma}}(\boldsymbol{F}, V)$ is the Cauchy stress tensor, $\hat{\boldsymbol{\sigma}}$ stands for the appropriate constitutive equation for path dependent materials (large strains elasto-plastic constitutive equations based on the multiplicative split [33] are used here, see Section 4), $\boldsymbol{F}$ is the total deformation gradient and $V$ represents the set of internal variables of the model. $\mathbf{u}_{0}$ stands for the initial displacement, $\mathbf{b}$ is the external body force vector and $\partial \Omega_{t}=\Gamma_{u} \cup \Gamma_{\bar{t}}$ $\left(\Gamma_{u} \cap \Gamma_{\bar{t}}=\emptyset\right)$ defines the boundary of the domain where displacements $\overline{\boldsymbol{u}}$ and tractions $\overline{\boldsymbol{t}}$ are prescribed.

The same problem may be restated using a mixed two-field displacement-pressure $(\mathbf{u}-p)$ formulation. Introducing a volumetric/deviatoric decomposition of the Cauchy stress tensor, the standard expression of the strong form of the equilibrium equation becomes

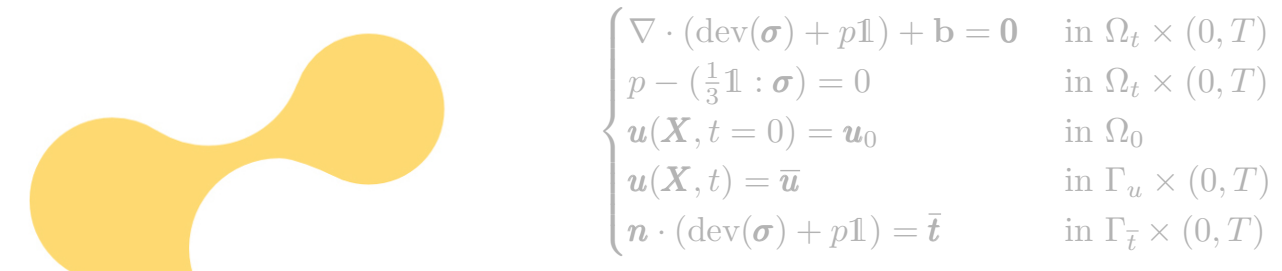

where $\operatorname{dev}(\sigma)$ is the deviatoric part of the Cauchy stress tensor, $p$ is the Cauchy pressure and $\mathbb{1}$ stands for the second order identity tensor.

A three-field displacen problem is also possible state, $\theta$, is introduced
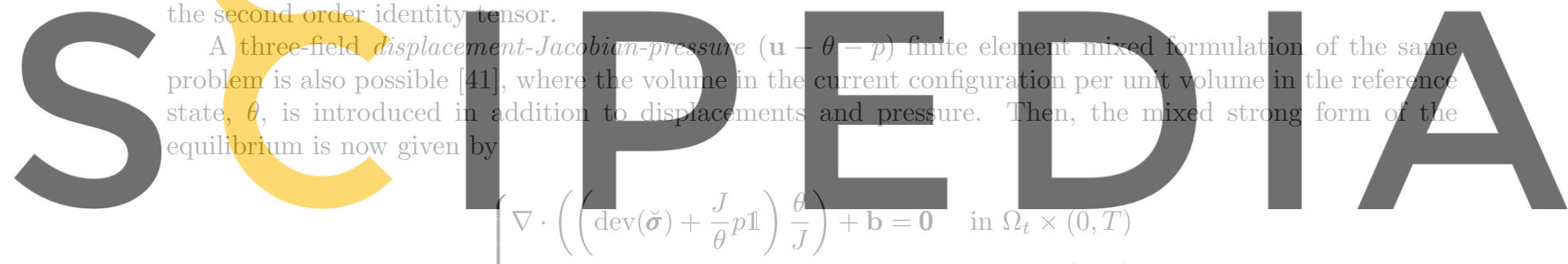

Register for free at https//www.sejpedia.com to download"the`version without the watermark

$$
\begin{cases}p-\left(\frac{1}{3} \mathbb{1}: \bar{\sigma}\right)=0 & \text { in } \Omega_{t} \times(0, T) \\ \boldsymbol{u}(\boldsymbol{X}, t=0)=\boldsymbol{u}_{0} & \text { in } \Omega_{0} \\ \boldsymbol{u}(\boldsymbol{X}, t)=\overline{\boldsymbol{u}} & \text { in } \Gamma_{u} \times(0, T) \\ \boldsymbol{n} \cdot\left(\left(\operatorname{dev}(\breve{\boldsymbol{\sigma}})+\frac{J}{\theta} p \mathbb{1}\right) \frac{\theta}{J}\right)=\overline{\boldsymbol{t}} & \text { in } \Gamma_{\bar{t}} \times(0, T)\end{cases}
$$

where $J=\operatorname{det}(\mathbf{F})$ is the determinant of the deformation gradient, $\theta$ is the volumetric deformation and $\breve{\boldsymbol{\sigma}}=\hat{\boldsymbol{\sigma}}(\breve{\boldsymbol{F}}, V)$ is the Cauchy stress evaluated with the assumed deformation gradient $\breve{\mathbf{F}}$. For this formulation, the second Piola-Kirchhoff stress tensor is related to the Cauchy stress tensor as:

$$
\breve{\boldsymbol{\sigma}}=\frac{1}{\theta} \breve{\boldsymbol{F}} \cdot \breve{S} \cdot \breve{\boldsymbol{F}}^{T}
$$

The assumed deformation gradient is defined as:

$$
\breve{\mathbf{F}}=\mathbf{F}^{v} \mathbf{F}^{d}=\left(\theta^{\frac{1}{3}}\right)\left(\operatorname{det}(\mathbf{F})^{-\frac{1}{3}} \mathbf{F}\right)=\left(\frac{\theta}{\operatorname{det}(\mathbf{F})}\right)^{\frac{1}{3}} \mathbf{F}
$$

That is, the deviatoric part of the deformation gradient, $\mathbf{F}^{d}$, is preserved whereas the volumetric part, $\mathbf{F}^{v}$, is replaced with the $\theta$ variable. Note that in this formulation the Cauchy stress tensor depends on both, 
displacements and on the Jacobian. Despite that, the usual form of strain-driven stress integration schemes are completely suitable for this formulation.

In plane strain conditions, the assumed deformation gradient, Equation (5), has to be redefined in order to guarantee that the out of plane component of the deformation is equal to unity [35]:

$$
\breve{\mathbf{F}}^{2 D}=\left(\frac{\theta}{\operatorname{det}(\mathbf{F})}\right)^{\frac{1}{2}} \mathbf{F}^{2 D}
$$

Then, the three dimensional constitutive law is evaluated with:

$$
\breve{\mathbf{F}}^{C L}=\left(\begin{array}{cc}
\breve{\mathbf{F}}^{2 D} & \overrightarrow{0}^{T} \\
\overrightarrow{0} & 1
\end{array}\right)
$$

The previous formulation adds two extra balance equations with respect to the displacement-based formulation. As it will be shown in Section 3.1.2, where the discrete Finite Element equations are presented, this fact results in the addition of two degrees of freedom per node $(\theta$ and $p)$ with respect to the primal formulation. It becomes unclear which of the two extra equations may be then responsible for any improvement on the volumetric locking effect. To clarify this point, a third mixed formulation, having only the displacement $\mathbf{u}$ and the volume deformation $\theta$ as independent variables (the $\mathbf{u}-\theta$ formulation), will also be assessed:
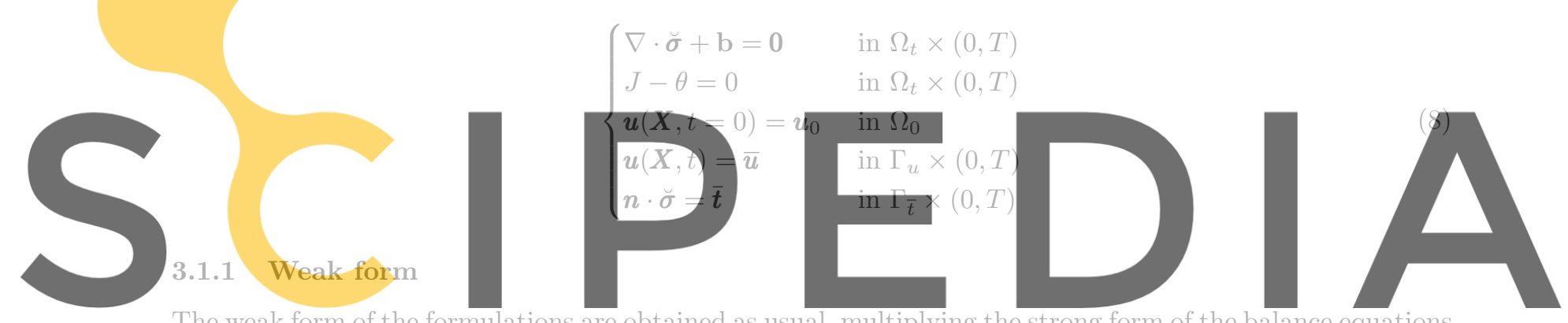

The weak form of the formulations are obtained as usual, multiplying the strong form of the balance equations for test functions and integrating over the entire domain. To show the expression in the most complex case,

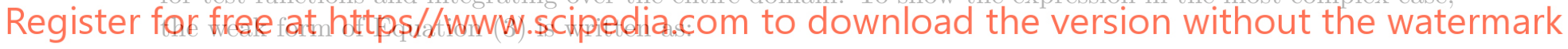

$$
\begin{cases}\int_{\Omega_{t}} \nabla \eta_{i k}\left(\mathbb{I}_{k l i j}^{d} \breve{\sigma}_{i j}+\frac{J}{\theta} p \delta_{k i}\right) \frac{\theta}{J} d \Omega_{t}=\int_{\Omega_{t}} \eta_{i} b_{i} d \Omega_{t}+\int_{\Gamma_{\bar{t}}} \eta_{i} \bar{t}_{i} d \Gamma & \forall \eta \in \mathrm{V} \\ \int_{\Omega_{t}} \zeta(J-\theta) \frac{1}{J} d \Omega_{t}=0 & \forall \zeta \in \mathrm{G} \\ \int_{\Omega_{t}} q\left(p-\frac{1}{3} \breve{\sigma}_{k k}\right) \frac{1}{J} d \Omega_{t}=0 & \forall q \in \mathrm{Q}\end{cases}
$$

where $\mathbb{I}_{k l i j}^{d}=\mathbb{I}_{k l i j}-\frac{1}{3} \delta_{l k} \delta_{i j}$ is the deviatoric projection tensor. Being $\eta \in \mathrm{V}, \zeta \in \mathrm{G}$ and $q \in \mathrm{Q}$ valued functions in the space of virtual displacements V, virtual volume Jacobians G, and virtual pressures $Q$, respectively.

\subsubsection{Finite element discrete equations}

In order to obtain the finite element discrete equations of the weak form of the mixed formulations, first the nodal variables are approximated with the FE shape functions

$$
\left\{\begin{array}{l}
\mathbf{u}^{h}=\mathbf{N}_{u} \cdot \tilde{\mathbf{u}} \\
\theta^{h}=\mathbf{N} \cdot \tilde{\boldsymbol{\theta}} \\
p^{h}=\mathbf{N} \cdot \tilde{\mathbf{p}}
\end{array}\right.
$$


where $\mathbf{u}^{h}, \theta^{h}$ and $p^{h}$ are finite element approximations of displacement, the Jacobian and the pressure whereas $\tilde{\mathbf{u}}, \tilde{\boldsymbol{\theta}}$ and $\tilde{\mathbf{p}}$ are the nodal values. The same order interpolation functions are used for scalar fields and displacements: $\mathbf{N}=\left[N_{1}, N_{2}, \ldots, N_{n}\right]$ and $\mathbf{N}_{u}=\left[N_{1} \mathbb{1}, N_{2} \mathbb{1}, \ldots, N_{n} \mathbb{1}\right]$ where $n$ is the number of nodes.

The matrix form of the Galerkin expression of the $\mathbf{u}-\theta-p$ formulation, Equation (3), is obtained introducing the previous approximations to the weak form, Equation (9):

$$
\left\{\begin{array}{l}
\mathbf{P}\left(\operatorname{dev}(\breve{\boldsymbol{\sigma}}) \frac{\theta}{J}\right)+\mathbf{Q} \cdot \tilde{\mathbf{p}}=\mathbf{f}^{e x t} \\
\mathbf{M} \cdot \tilde{\boldsymbol{\theta}}=\mathbf{f}^{\theta} \\
\mathbf{M} \cdot \tilde{\mathbf{p}}=\mathbf{f}^{p}(\breve{\boldsymbol{\sigma}})
\end{array}\right.
$$

where the matrices and vectors of Equation (11) are defined as

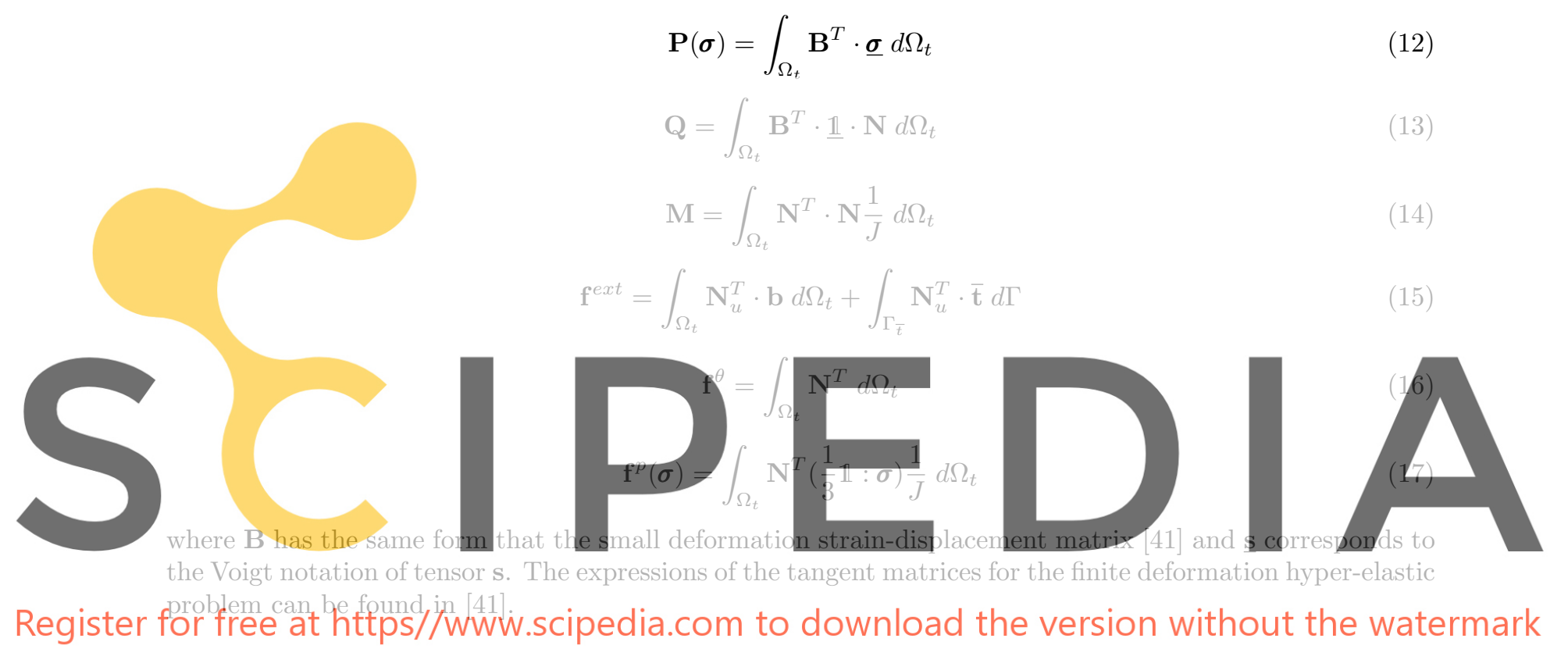

\subsubsection{Stabilization of the mechanical problem}

In our approach, linear shape functions are used for all the variables of the mixed formulations. The stabilization method used is the so-called Polynomial Pressure Projection (PPP) previously applied to stabilize Stokes equations $[4,11]$. The PPP has two main ingredients:

1. A mixed equal order interpolation of the scalar and vector fields.

2. A $L_{2}$ projection of the scalar variables (volume or pressure variables).

The method is obtained by modifying the mixed variational equation (i.e. the pressure continuity equation) by using local $L_{2}$ polynomial pressure projections of the pressure variable. The application of the projections in conjunction with minimization of the problem field mismatch, eliminates the inconsistency of equal-order approximations and leads to a stable variational formulation. Unlike other stabilization methods, the Polynomial Pressure Projection does not require specification of a mesh dependent stabilization parameter or calculation of higher-order derivatives. It uses a projection on a discontinuous space and, as a consequence, can be implemented at element level avoiding the need of mesh or problem dependent parameters. The implementation of this stabilization scheme reduces to a simple modification of the weak continuity equation (the incompressibility constraint).

Given a function $p \in L_{2}$, the $L_{2}$ projection operator $\breve{p}: L_{2} \rightarrow Q^{0}$ is defined by 


$$
\int_{\Omega_{0}} \breve{q}(p-\breve{p}) d \Omega_{0}=0 \quad \forall \breve{q} \in Q^{0}
$$

where $\breve{p}$ is the best approximation of the pressure $p$ in the space of polynomials of order $\mathcal{O}\left(Q^{0}\right)$.

To stabilize the mixed forms given by Equations (2), (3) and (8) we add at each element the projection operator to the scalar continuity equations.

$$
\int_{\Omega_{0}^{e}}(q-\breve{q}) \frac{\alpha_{s}}{\mu}(p-\breve{p}) d \Omega_{0}=0
$$

where $\alpha_{s}$ is the stabilization parameter and $\mu$ is the material shear modulus.

Then, the discrete and stabilized finite element equations of the $\mathbf{u}-\theta-p$ formulation, Equations (3) and (11), are written as:

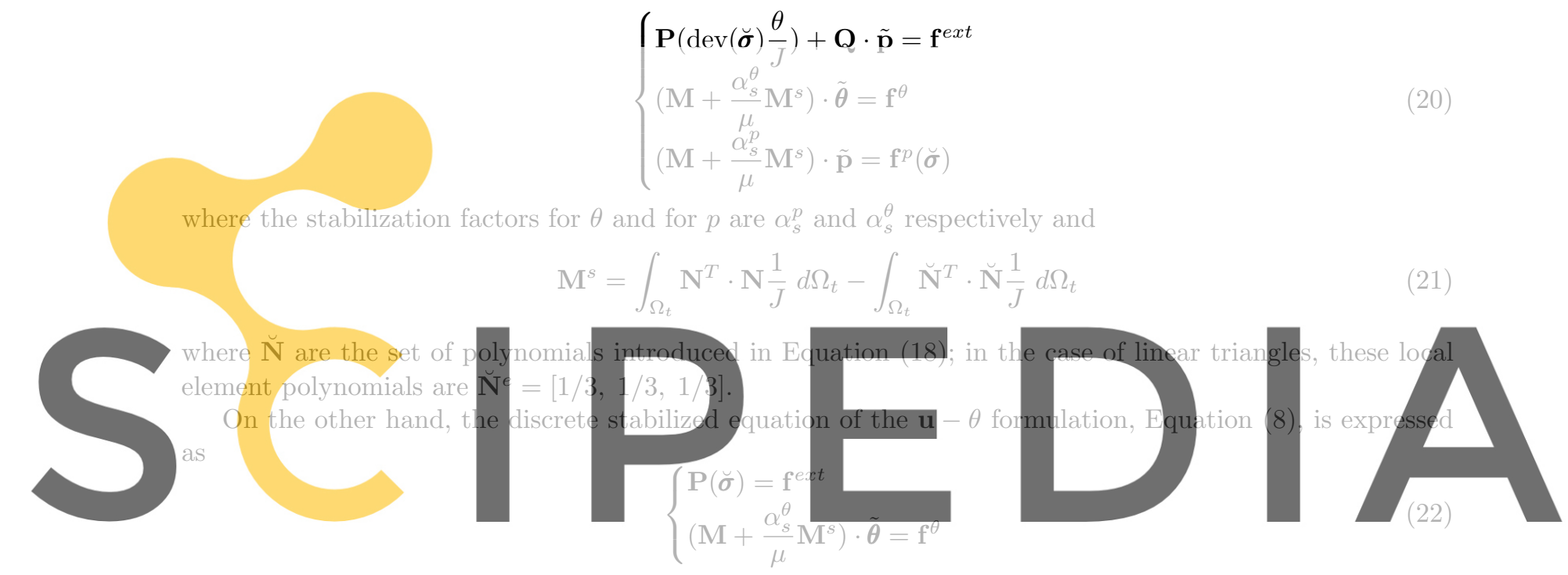

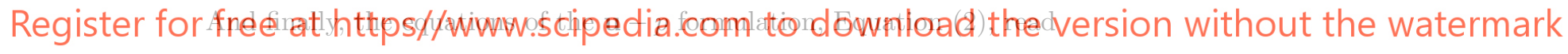

$$
\left\{\begin{array}{l}
\mathbb{P}(\operatorname{dev}(\sigma))+\mathrm{Q} \cdot \tilde{\mathrm{p}}=\mathrm{f}^{e x t} \\
\left(\mathbf{M}+\frac{\alpha_{s}^{p}}{\mu} \mathbf{M}^{s}\right) \cdot \tilde{\mathbf{p}}=\mathbf{f}^{p}(\boldsymbol{\sigma})
\end{array}\right.
$$

\subsection{Fluid-saturated multiple phase porous media}

The balance of mass and linear momentum equations for multiple-phase deformable porous media using a displacement-water pressure $\left(\mathbf{u}-p_{w}\right)$ formulation in quasi-static cases, may be written in the current deformed configuration as:

$$
\begin{cases}\nabla \cdot \boldsymbol{\sigma}+\mathbf{b}=\mathbf{0} & \text { in } \Omega_{t} \times(0, T) \\ \frac{-1}{\kappa_{w}} \dot{p}_{w}+\nabla \cdot \mathbf{v}+\nabla \cdot \mathbf{v}^{d}=0 & \text { in } \Omega_{t} \times(0, T) \\ \boldsymbol{u}(\boldsymbol{X}, t=0)=\boldsymbol{u}_{0} & \text { in } \Omega_{0} \\ p_{w}(\boldsymbol{X}, t=0)=p_{w_{0}} & \text { in } \Omega_{0} \\ \boldsymbol{u}(\boldsymbol{X}, t)=\overline{\boldsymbol{u}} & \text { in } \Gamma_{u} \times(0, T) \\ \boldsymbol{n} \cdot \boldsymbol{\sigma}=\overline{\boldsymbol{t}} & \text { in } \Gamma_{\bar{t}} \times(0, T) \\ p_{w}(\boldsymbol{X}, t)=\bar{p}_{w} & \text { in } \Gamma_{p_{w}} \times(0, T) \\ -\boldsymbol{n} \cdot \boldsymbol{v}^{d}=\bar{g} & \text { in } \Gamma_{g} \times(0, T)\end{cases}
$$


where $p_{w}$ is the Cauchy water pressure, $\left(\dot{p}_{w}=\frac{d p_{w}}{d t}\right)$ is the material time derivative with respect to the solid phase, $\kappa_{w}$ is the water compressibility and $\mathbf{v}^{d}$ is the Darcy's velocity. The boundary of the domain is divided in two parts, $\partial \Omega_{t}=\Gamma_{p_{w}} \cup \Gamma_{g}\left(\Gamma_{p_{w}} \cap \Gamma_{g}=\emptyset\right)$, where fixed water pressure $\bar{p}_{w}$ and prescribed water flow $\bar{g}$ are imposed. The interested reader is referred to $[5,16,36]$ for further information.

According to the principle of effective stress, the total stress tensor, $\boldsymbol{\sigma}$, is equal to the sum of the pore pressure, $p_{w}$, and the effective stress, $\boldsymbol{\sigma}^{\prime}$ :

$$
\sigma=\sigma^{\prime}+p_{w} \mathbb{1}
$$

and the effective stress, which only depends on the strains of the solid skeleton, is defined as

$$
\boldsymbol{\sigma}^{\prime}=\hat{\boldsymbol{\sigma}}^{\prime}(\boldsymbol{F}, V)=\operatorname{dev}\left(\boldsymbol{\sigma}^{\prime}\right)+p^{\prime} \mathbb{1}
$$

Two different mixed formulations for the coupled poromechanics problem are explored for use in PFEM. The first one is the displacement-effective pressure-water pressure, which is expressed in the strong form as follows:

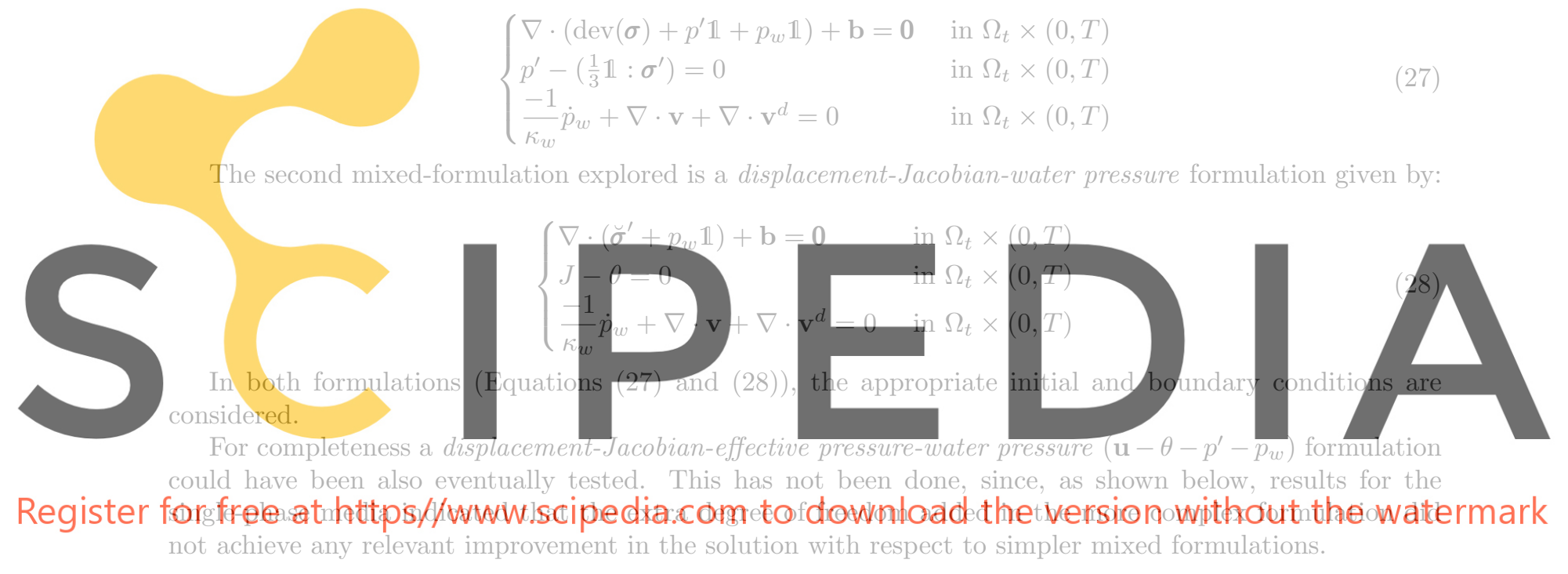

\subsubsection{Finite element discrete equations}

After obtaining the weak form of the problem, the semi-discrete equations of the primal formulation, Equation (24), are given by:

$$
\left\{\begin{array}{l}
\mathbf{P}\left(\boldsymbol{\sigma}^{\prime}\right)+\mathbf{Q} \cdot \tilde{\mathbf{p}}_{w}=\mathbf{f}^{e x t} \\
\mathbf{Q}^{\star T} \cdot \dot{\tilde{\mathbf{u}}}-\frac{1}{\kappa_{w}} \mathbf{M} \cdot \dot{\tilde{\mathbf{p}}}_{w}-\mathbf{H} \cdot \tilde{\mathbf{p}}_{w}=\mathbf{f}^{p_{w}}
\end{array}\right.
$$

where the water pressure is approximated as $p_{w}^{h}=\mathbf{N} \cdot \tilde{\mathbf{p}}_{w}$, the constitutive equation of the Darcy flow -described in Section 4- has been introduced and

$$
\begin{gathered}
\mathbf{Q}^{\star T}=\int_{\Omega_{t}} \mathbf{N}^{T} \cdot \underline{\mathbb{1}} \cdot \mathbf{B} \frac{1}{J} d \Omega_{t} \\
\mathbf{H}=\int_{\Omega_{t}}(\nabla \mathbf{N})^{T} \cdot \mathbf{k}_{p} \cdot(\nabla \mathbf{N}) \frac{1}{J} d \Omega_{t} \\
\mathbf{f}^{p_{w}}=\int_{\Omega t}(\nabla \mathbf{N})^{T} \cdot \mathbf{k}_{p} \cdot \mathbf{g} \frac{\rho_{w}}{J} d \Omega_{t}+\int_{\Gamma_{g}}(\nabla \mathbf{N})^{T} \bar{g} \frac{1}{J} d \Gamma
\end{gathered}
$$

where $\mathbf{g}$ is the gravity. 


\subsubsection{Stabilization of the mass conservation equation}

The monolithic approach of the hydromechanical problem, Equation (29), fails to satisfy the inf-sup condition in the undrained limit if equal order interpolants are used for both displacement and water pressure [22]. Several techniques have been developed to address this problem introducing a characteristic stabilization parameter which depends on the element size and/or material properties, see [23]. The fluid pressure Laplacian stabilization (FPL) technique is used in this work because of its good solution accuracy and of the symmetry of the resulting system.

Using FPL-stabilization, a term is added to the weak form of the mass balance equation of the hydromechanical problem:

$$
\int_{\Omega_{t}} \tau \nabla q \cdot \mathbf{k}_{p} \cdot \nabla \dot{p}_{w} \frac{1}{J} d \Omega_{t}=0 \quad \forall q \in Q
$$

where $\mathbf{k}_{p}$ is the permeability tensor and $\tau$ is the stabilization parameter. The expression chosen for the $\tau$ is the one proposed by Preisig et al. in [23].

Then, the stabilized semi-discrete equations of the primal formulation, Equation (24), are given by

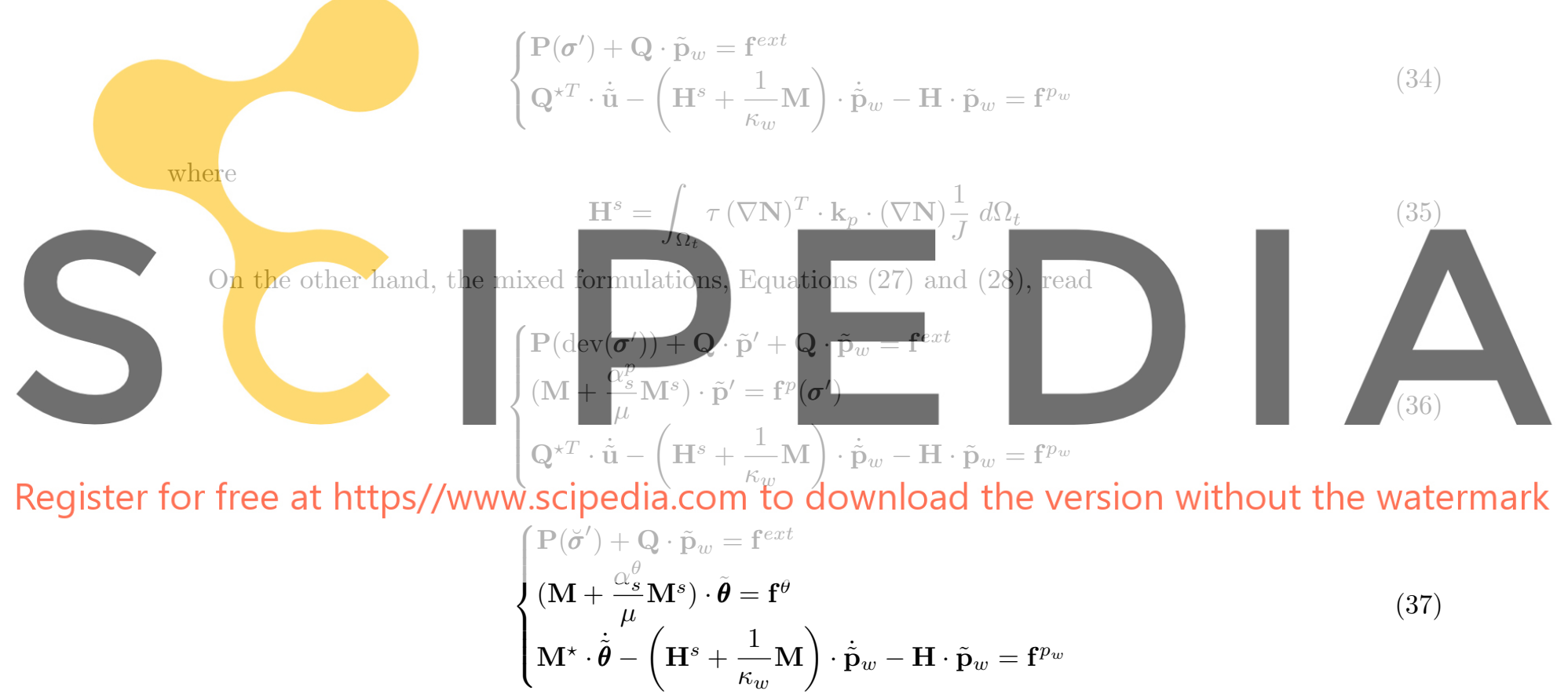

where $p^{\prime h}=\mathbf{N} \cdot \tilde{\mathbf{p}}^{\prime}$ and

$$
\mathbf{M}^{\star}=\int_{\Omega_{t}} \mathbf{N}^{T} \cdot \mathbf{N} \frac{1}{\theta J} d \Omega_{t}
$$

As before PPP stabilization of the scalar balance equation is applied in the $\mathbf{u}-p^{\prime}-p_{w}$ and $\mathbf{u}-\theta-p_{w}$ formulations. In addition, the mass balance equation is also stabilized in all cases, using the procedure that is described above.

These equations are solved with a monolithic approach and an implicit time integration scheme is used. That is, the derivative of the water pressure is approximated as $\dot{\tilde{\mathbf{p}}}_{w} \approx \frac{\Delta \tilde{\mathbf{p}}_{w}}{\Delta t}=\frac{\tilde{\mathbf{p}}_{w}^{t+\Delta t}-\tilde{\mathbf{p}}_{w}^{t}}{\Delta t}$ and the velocity of the solid skeleton is $\dot{\tilde{\mathbf{u}}} \approx \frac{\Delta \tilde{\mathbf{u}}}{\Delta t}=\frac{\tilde{\mathbf{u}}^{t+\Delta t}-\tilde{\mathbf{u}}^{t}}{\Delta t}$. On the mixed $\mathbf{u}-\theta-p_{w}$ formulation the following relation is used $\nabla \cdot \mathbf{v}=\frac{\dot{J}}{J} \approx \frac{\dot{\theta}^{h}}{\theta^{h}}$. 


\section{Constitutive relations}

In this work, large strains elasto-plastic constitutive equations are employed [33]. These models are formulated using a multiplicative split of the deformation gradient into an elastic and a plastic part. To this end, an intermediate configuration of irreversible (plastic) deformations is introduced, relative to which the elastic response of the material is characterized. As a consequence, the deformation gradient is decomposed as:

$$
\boldsymbol{F}=\boldsymbol{F}^{e} \cdot \boldsymbol{F}^{p}
$$

In formulations that consider the Jacobian as a nodal variable, the assumed deformation gradient also splits multiplicatively in a plastic and elastic part, $\breve{\boldsymbol{F}}=\breve{\boldsymbol{F}}^{e} \cdot \breve{\boldsymbol{F}}^{p}$. In these formulations, as mentioned before, the local problem retains a strain-driven format. Therefore, the same procedures and constitutive relations as the displacement-based finite element are used. However, in these cases, a different definition of the deformation is employed. The rest of the equations defining the local problem can be found in [32].

Two types of constitutive relations are used, one for the purely mechanical problem (total-stress analysis) and another one for the coupled hydro-mechanical problem. For the so-called total stress analysis, the saturated soil is assumed to satisfy a Tresca yield criterion:

$$
f(\boldsymbol{\tau})=J_{2} \cos \left(\theta_{L}\right)-S_{u}
$$

where $\boldsymbol{\tau}=J \boldsymbol{\sigma}$ is the Kirchhoff stress tensor, $J_{2}$ is the second invariant of the Kirchhoff stress, $\theta_{L}$ is the Lode Angle and $S_{u}$ is the undrained shear strength, a characteristic parameter of the soil. The sharp edges of the yield surface are smoothed with a $\mathrm{C} 2$ approximation [1]. The elastic regime is assumed to fulfil a linear model between the Kirchhoff stress and the elastic Hencky Strain:

$$
\boldsymbol{\tau}=J p \mathbb{1}+\operatorname{dev}(\boldsymbol{\tau})=K \epsilon_{v}^{e} \mathbb{1}+2 G \boldsymbol{\epsilon}_{d}^{e}
$$

where $\boldsymbol{\epsilon}^{e}=\ln \left(\boldsymbol{b}^{e}\right) / 2$ is the elastic Hencky Strain and $\boldsymbol{b}^{e}=\boldsymbol{F}^{e} \cdot \boldsymbol{F}^{e T}$ the elastic left Cauchy-Green tensor. $\epsilon_{v}^{e}=\mathbb{1}: \boldsymbol{\epsilon}^{e}=\ln \left(J^{e}\right)$ and $\boldsymbol{\epsilon}_{d}^{e}=\mathbb{I}^{d}: \boldsymbol{\epsilon}^{e}=\ln \left(\overline{\boldsymbol{b}}^{e}\right) / 2$ are the volumetric and the deviatoric part of the elastic Hencky strain, respectively, and $\overline{\boldsymbol{b}}^{e}=J^{e-2 / 3} \boldsymbol{b}^{e}$ is the deviatoric part of the elastic Left Cauchy Green tensor. $K$ and $G$ are the bulk and shear modulus of the material.

For the coupled hydro-mechanical analysis, a Modified Cam Clay model is used [6, 29]. The elastic part of the model follows an hyperelastic model proposed by Houlsby [13]; then, the volumetric and deviatoric part of the effective Kirchhoff stress, $\boldsymbol{\tau}^{\prime}=\pi^{\prime} \mathbb{1}+\boldsymbol{\tau}_{d}$, are computed according to:

$$
\left\{\begin{array}{l}
\pi^{\prime}=-p_{0} \exp \left(\frac{-\epsilon_{v}^{e}}{\kappa^{*}}\right)\left(1+\frac{\alpha}{\kappa^{*}}\left\|\epsilon_{d}^{e}\right\|^{2}\right) \\
\boldsymbol{\tau}_{d}=2\left(G_{0}+\alpha p_{0} \exp \left(\frac{-\epsilon_{v}^{e}}{\kappa^{*}}\right)\right) \epsilon_{d}^{e}
\end{array}\right.
$$

where $\kappa^{*}=\frac{\kappa}{1+e_{0}}, \kappa$ is the slope of the swelling line, $e_{0}$ is the initial void ratio, $G_{0}$ is the constant part of the shear modulus, $\alpha \geqslant 0$ is a parameter and $p_{0}$ is a reference pressure.

Note that if $\alpha>0$, the volumetric and deviatoric elastic behavior is coupled: the bulk modulus, $K=$ $\frac{d \pi^{\prime}}{d \epsilon_{v}}=\frac{p_{0}}{\kappa^{*}} \exp \left(\frac{-\epsilon_{v}^{e}}{\kappa^{*}}\right)\left(1+\frac{\alpha}{\kappa^{*}}\left\|\epsilon_{d}^{e}\right\|^{2}\right)=\frac{-\pi^{\prime}\left(\epsilon_{v}^{e}, \epsilon_{d}^{e}\right)}{\kappa^{*}}$, varies with the elastic distortional and volumetric strains whereas the shear modulus, $G=G_{0}+\alpha p_{0} \exp \left(\frac{-\epsilon_{v}^{e}}{\kappa^{*}}\right)$, depends on the volumetric elastic strain; in fact, coupling terms appear on the elastic stiffness matrix.

The problem is completed with the yield surface and the hardening law defined by:

$$
\begin{gathered}
f\left(\boldsymbol{\tau}^{\prime}\right)=\left(\frac{\sqrt{3} J_{2}}{M\left(\theta_{L}\right)}\right)^{2}+\pi^{\prime}\left(\pi^{\prime}-p_{c}\right) \\
p_{c}=p_{c_{0}} \exp \left(\frac{-\epsilon_{v}^{p}}{\lambda^{*}-\kappa^{*}}\right)
\end{gathered}
$$


where $M$ is the slope of the Critical state line in the $\left(\pi^{\prime}-\sqrt{3} J_{2}\right)$ plane and may be made dependent of the Lode's Angle $\theta_{L}, p_{c}$ is the isotropic preconsolidation pressure, $\lambda^{*}=\frac{\lambda}{1+e_{0}}, \lambda$ is the slope of the virgin consolidation line and the reference preconsolidation pressure is denoted by $p_{c_{0}}$.

In both models associated plasticity is assumed. The integration of the elasto-plastic constitutive relations is performed with an explicit scheme with adaptive sub-stepping; correction for the yield surface drift is applied [18, 34].

A large deformation generalization of the Darcy's Law, excluding inertial effects is used:

$$
\mathbf{v}^{d}=\mathbf{k}_{p} \cdot\left(\nabla p_{w}+\rho_{w} \mathbf{g}\right)
$$

where $\mathbf{k}_{p}=\frac{k_{p}^{\prime}}{g \rho_{w}} \mathbb{1}, k_{p}^{\prime}$ is the spatial permeability with velocity units and $\mathbf{g}$ is the gravity; the spatial description of the permeability tensor is assumed to be constant [16].

\section{$5 \quad$ Numerical examples}

In this section, a set of examples of increasing numerical complexity are employed to assess the performance of the different mixed formulations presented above in PFEM. An effort is made to separate effects due to the specific choice of mixed formulation from issues that are related to other aspects of PFEM. The first example involves on the indentation of a rigid-strip footing into a single-phase incompressible material (representing an undrained soil). In the second example, a flexible circular footing resting in a Modified Cam Clay soil is used to assess the two-phase formulations; a wide range of loading conditions -ranging from drained to undrained- are used. Finally, the method is applied to the modelling of a more challenging geo-mechanical problem: a Cone Penetration Test with pore pressure measurement $(\mathrm{CPTu})$.

\subsection{Rigid footing resting on a one-phase deformable media}

In this example, a rough rigid strip footing is pushed into a weightless Tresca soil. Footing displacement is imposed using Dirichlet boundary conditions. Due to the symmetry of the problem, only half of the problem is computed. The geometry of the domain (initial and final mesh) is depicted in Figure 2. Geometry and constitutive parameters $\left(E=100 \mathrm{kPa}, \nu=0.495\right.$ and $S_{u}=1 \mathrm{kPa}$; resulting in a rigidity index, $I_{r}=G / S_{u}$, of 33), are identical to those used by [14]. These authors analyzed this problem using an ALE (Arbitrary Lagrangian-Eulerian) method, investigating the effect of different high-order elements on the primal formulation.

Figure 3 presents curves of normalized settlement vs normalized soil resistance for the three stabilized formulations. The normalized limit resistance provides a bearing capacity factor for this problem, $N_{c}=q / S_{u}$, where $q$ is the vertical stress applied by the footing and $S_{u}$ the strength of the soil. A reference solution from [14] (obtained with 21-noded elements) is also included for comparison purposes.

It is clear that all the solutions obtained with stabilized formulations match the reference solution. The oscillations in these curves result from errors introduced at remeshing events and are independent of the mixed formulation employed. Indeed, the sudden drops in resistance are related to the size of the elements close to the rigid footing. With the mesh adaptive procedures of PFEM nodes initially belonging to the contour of the domain are kept at the contour and its position is never modified. However, to maintain accuracy, new nodes may be inserted when the distance between two adjacent nodes at the contour becomes to large, or a node may be deleted when it comes too close to another node. In this problem, the last node with prescribed displacements (representing the footing corner) and the first node without restrictions (corresponding to the soil adjacent to the corner) tend to separate. The insertion of a new node in the middle of this segment causes the periodic drops in the resistance curves.

The effect of the different mixed formulations is more visible in the vertical profiles of stresses presented in Figure 4(a). Stresses obtained with the $\mathbf{u}-\theta-p$ and $\mathbf{u}-\theta$ formulations show smaller scatter than those resulting from the $\mathbf{u}-p$ formulation show more. This is even clearer when the stress invariants alongside the same vertical are plotted (see Figure 4(b)); the main differences affect the Lode angle, that it is almost 


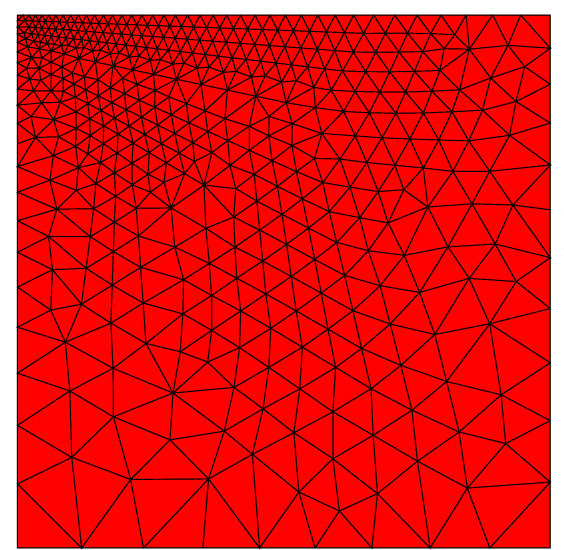

(a)

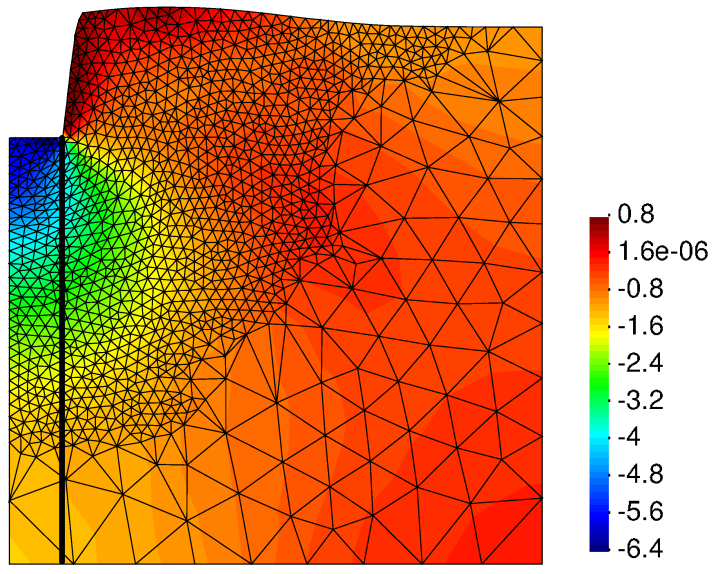

(b)

Figure 2: Rigid Footing test. $I_{r}=33$. Initial and final finite element mesh with indication of the position of the vertical profile. Contour plot of the pressure $(\mathrm{kPa})$.

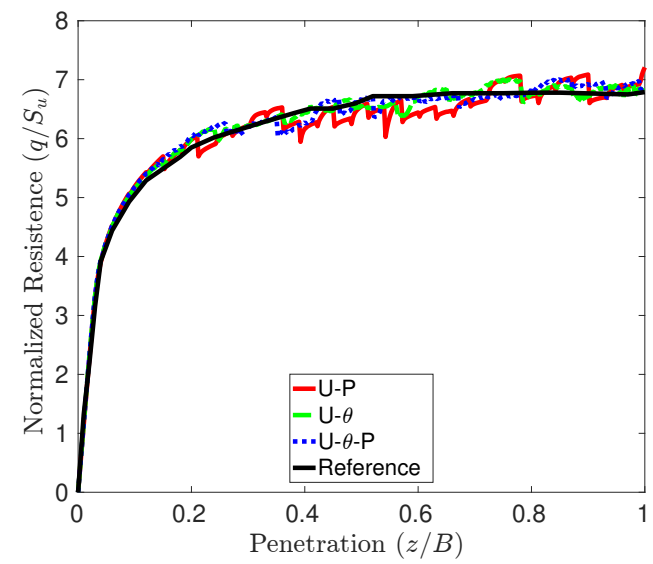

Figure 3: Rigid Footing test. $I_{r}=33$. Normalized load-displacement curve for different formulations along with the reference solution of Kardani et al [14].

coincident for the two formulations that have the Jacobian as a nodal variable. In contrast, in the formulation that does not have the Jacobian as a nodal variable, the Lode angle shows a large scatter, particularly on the upper $2 \mathrm{~m}$, where the plastic region is located. Due to the definition of the yield surface, the scatter in Lode angle explains also the variations of the second stress invariant $\left(J_{2}\right)$ in the same region.

It may be questioned if the stress scatter noticed above is due solely to the choice of mixed formulation. To clarify this aspect a further analysis has been performed. The geometry and definition of the problem are the same, but now the soil is considered more rigid $\left(E=1495 \mathrm{kPa}, \nu=0.495, S_{u}=1 \mathrm{kPa}\right.$ resulting in $\left.I_{r}=500\right)$, so that failure will be reached at lower displacements. Also, mesh refinement is disabled.

Figure 5 shows the mesh at the end of computations; since displacements are small, the mesh is not distorted, despite the achievement of a clear limit load (at a footing penetration $z / B=0.02$; see Figure 6 ). As before, the differences in normalized resistance between the formulations are small, with the $\mathbf{u}-p$ formulation indicating a slightly higher $(2 \%)$ limit load. More importantly perhaps, the vertical profiles of stress components and invariants, Figure 7, show unequivocally that including the Jacobian as a nodal 


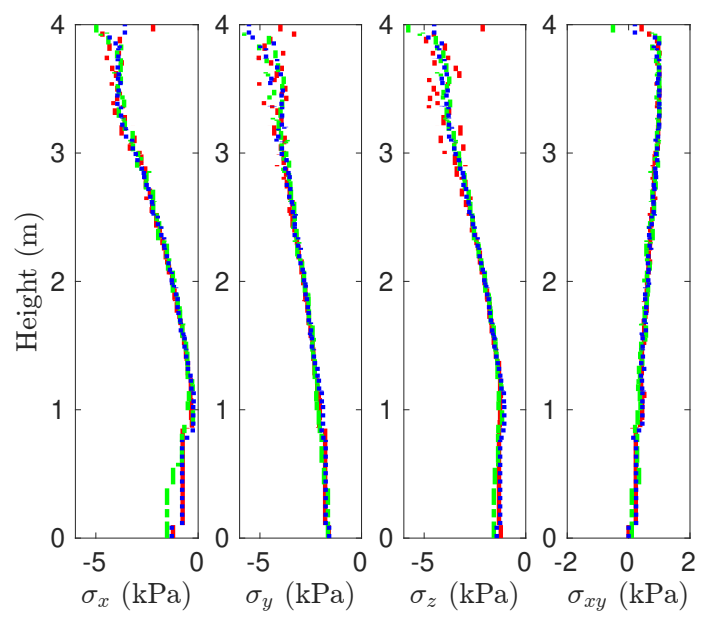

(a)
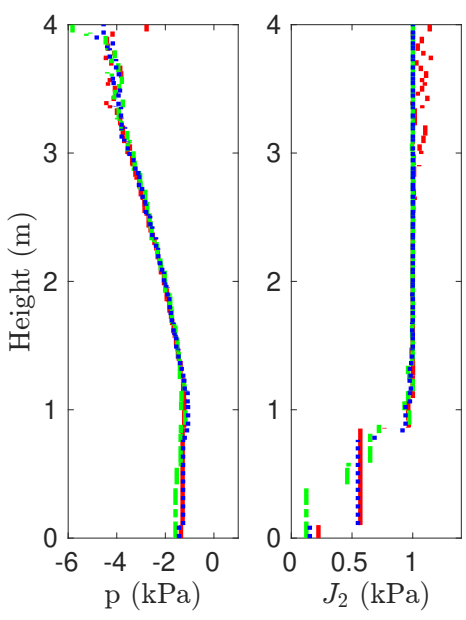

(b)

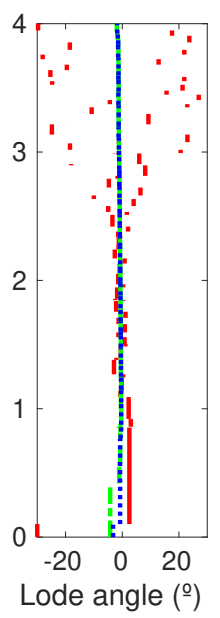

Lode angle $(\stackrel{\circ}{)}$

Figure 4: Rigid Footing test. $I_{r}=33$. Stress state along the vertical line indicated in Figure 2 at a penetration depth of $z / B=1$; Cartesian components of the Cauchy stress (a) and stress invariants (b). $\mathbf{u}-p$ (red), $\mathbf{u}-\theta$ (green) and $\mathbf{u}-\theta-p$ (blue).

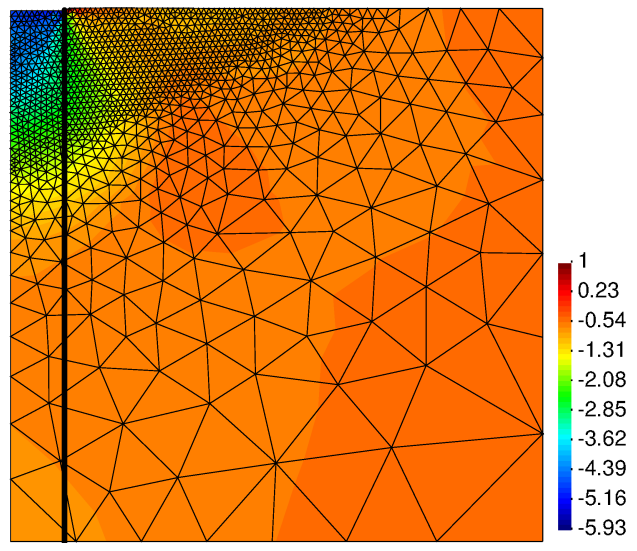

Figure 5: Rigid Footing test. $I_{r}=500$. Final finite element mesh with indication of the position of the vertical profile. Contour plot of the pressure $(\mathrm{kPa})$.

variable practically eliminates the scatter on computed Lode angle. Although in this problem such scatter has not caused significant problems at the global scale, this may not always be the case.

\subsection{Flexible circular footing resting on a two-phase deformable media}

The various two-phase formulations are examined using a problem that involves a flexible circular footing loading in a compressible clay. A similar geometry to the previous example is used; however, this case is axisymmetric and the footing is discretized with a load boundary condition. The model has a $0.25 \mathrm{~m}$ thick layer of elastic material placed on the top of the domain in order to avoid the problems that arise in the Modified Cam Clay model when the stress state approaches zero effective pressure. This layer of elements is always discretized using $\mathbf{u}-p_{w}$ elements. The initial stress state is obtained using a value of the coefficient of lateral stress, $K_{0}=0.5$. The problem and the values of the constitutive parameters (Table 1) are similar 


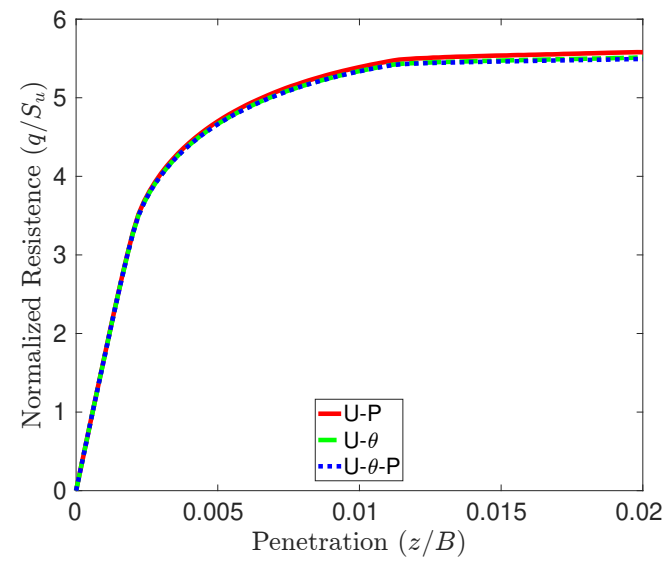

Figure 6: Rigid Footing test. $I_{r}=500$. Normalized load-displacement curve for different formulations.

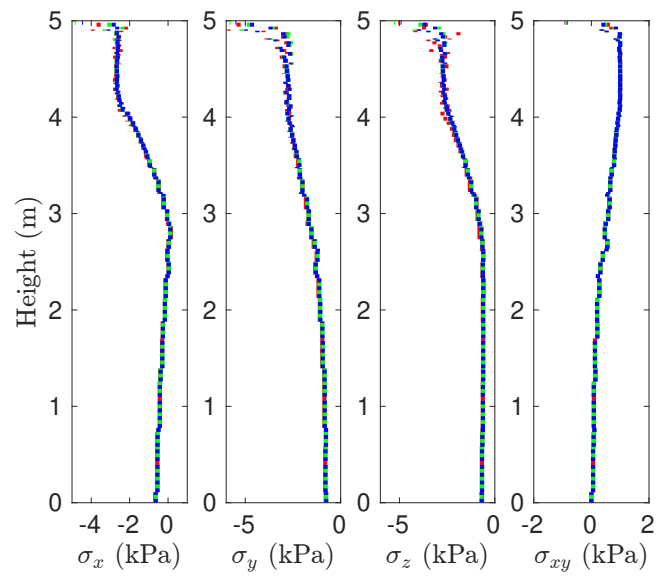

(a)
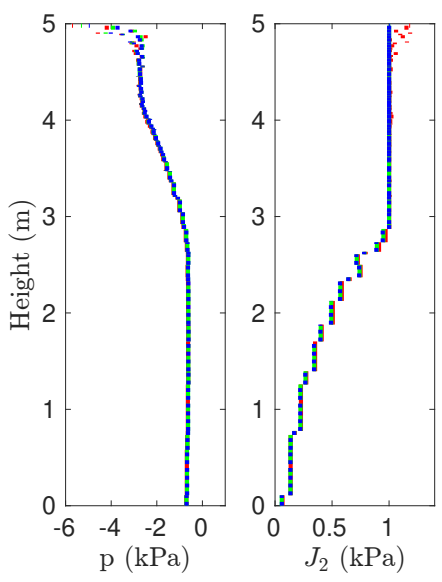

(b)

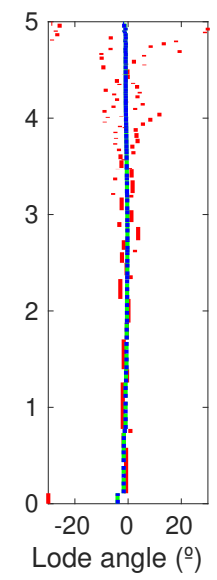

Lode angle (ํㅜ)

Figure 7: Rigid Footing test. $I_{r}=500$. Stress state along the vertical line indicated in Figure 5 at a penetration depth of $z / B=0.02$; Cartesian components of the Cauchy stress (a) and stress invariants (b). $\mathbf{u}-p$ (red), $\mathbf{u}-\theta$ (green) and $\mathbf{u}-\theta-p$ (blue).

to the ones presented in [7]; although they were using plane strain conditions and the initial stress state was obtained differently.

A vertical load of $100 \mathrm{kPa}$ is applied at a constant rate over a period of time; afterwards, this load is held constant to allow consolidation. Three different loading times $\left(T_{l}\right)$ are used: $0.001,10$ and 1000 days. The computational time-step is $\Delta t=T_{l} / 25$.

Figure 8 depicts the vertical displacement at the centerline of the footing as a function of the normalized time, $t / T_{l}$. For the fastest and slowest loading rates displacements cease to increase once the loading phase ends. Loading at the intermediate rate shows initial stiffness similar to the fast case and final settlement similar to the slow case. Figure 8 also plots the variation of water pressure at a point located below the footing center at a depth equal to the footing diameter. For the fastest loading rate, water pressure increases until the end of the loading phase; then remains constant. At the slowest loading rate no excess of pore pressure is generated, although a small hydrostatic increase (from $10 \mathrm{kPa}$ at the initial state to $10.30 \mathrm{kPa}$ at the end of the problem) is computed due to the settlement of the observation point. For the intermediate 
Table 1: Constitutive parameters adopted for the flexible circular footing example

\begin{tabular}{llllllllllll}
\hline & $\rho_{m}\left(\mathrm{~kg} / \mathrm{m}^{3}\right)$ & $\kappa^{*}$ & $p_{0}(\mathrm{kPa})$ & $\alpha$ & $G_{0}(\mathrm{kPa})$ & $\lambda^{*}$ & OCR & $\mathrm{M}$ & $k_{p}^{\prime}(\mathrm{m} / \mathrm{s})$ & $\mathrm{E}(\mathrm{kPa})$ & $\nu$ \\
\hline MCC & $2 \cdot 10^{3}$ & 0.05 & 10.0 & 0.0 & 200.0 & 0.10 & 1.5 & 1.0 & $8.64 \cdot 10^{-4}$ & - & - \\
Elastic & $2 \cdot 10^{3}$ & - & - & - & - & - & - & - & 1.0 & 1000 & 0.0 \\
\hline
\end{tabular}
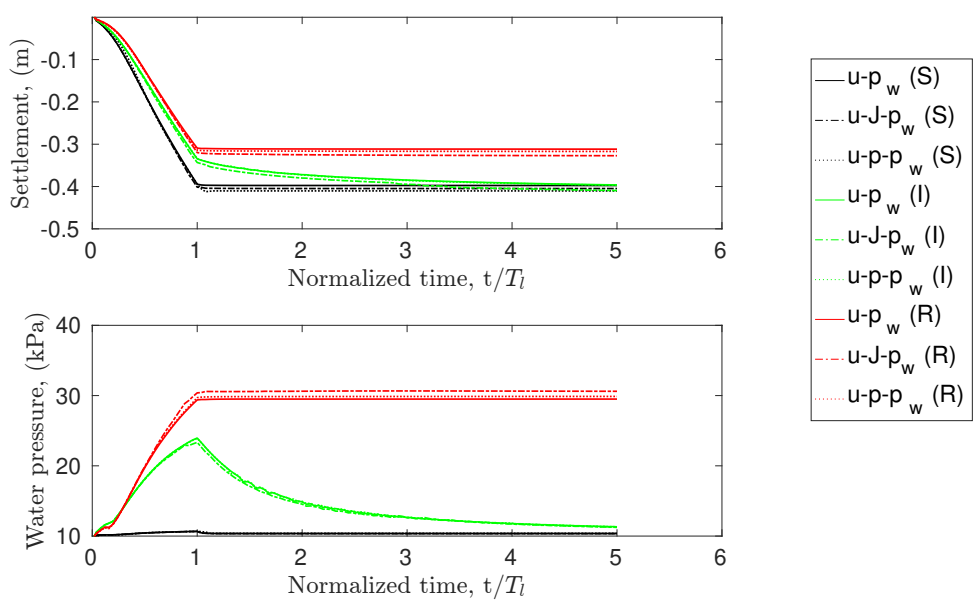

Figure 8: Hydromechanical footing. Evolution of displacements at the centerline (on top) and water pressure beneath the footing (bottom) for the three loading rates: Rapid (R), Intermediate (I) and Slow (S).

loading velocity the water pressure increases initially, attains a maximum at the end of loading and then decays.

In summary, the response is fully undrained in the fast case, fully drained in the slowest case and shows some consolidation at the intermediate loading rate. For the three loading rates there is little discrepancy between the solutions computed with the different formulations. The $\mathbf{u}-p_{w}$ formulation predicts slightly smaller settlements and (at the faster loading rate) smaller pore pressure generation.

\subsection{Cone penetration test}

The cone penetration test $(\mathrm{CPTu})$ is one of the most widely used in situ geotechnical testing methods. During the test an instrumented cone is pushed into the ground at a controlled rate. Tip resistance and sleeve friction are always recorded, and, very frequently, the pore water pressure just behind the cone tip $\left(u_{2}\right.$ position, see Robertson [27]) is also measured. From these measurements, stratigraphy and constitutive soil parameters are estimated based mostly on empirical correlations.

A CPTu in a Modified Cam Clay soil is performed. The cone is assumed rigid and smooth; contact between cone and soil is enforced using a Penalty method. Soil parameters are listed in Table 2; these values and the geometry of the problem try to mimic the case examined by Sheng et al using an ALE method [31].

The soil is weightless and the initial stress state imposed is given by $\sigma_{v}^{\prime}=-57.5 \mathrm{kPa}$ and $\sigma_{h}^{\prime}=-28.9$ $\mathrm{kPa}$. At the beginning of the computation the cone is wished-in-place with the tip at a depth of 2.8 cone radii. The cone is advanced downwards at $2 \mathrm{~cm} / \mathrm{s}$; a parametric analysis performed by Sheng et al. [31] indicates that undrained conditions will prevail at that velocity.

Net cone tip resistance, $q_{c}$, computed adding vertical forces from nodes at the cone tip and subtracting the initial vertical stress, is represented against normalized penetration in Figure 9. Pore water pressure at the $u_{2}$ position -computed interpolating from boundary nodes closer to the cone shoulder point- is shown in 
Table 2: Constitutive parameters adopted for the Cam Clay in the CPT example

\begin{tabular}{llllllll}
\hline$\kappa^{*}$ & $p_{0}(\mathrm{kPa})$ & $\alpha$ & $G_{0}(\mathrm{kPa})$ & $\lambda^{*}$ & $p_{c 0}(\mathrm{kPa})$ & $\mathrm{M}$ & $k_{p}^{\prime}(\mathrm{m} / \mathrm{s})$ \\
\hline 0.016 & 10.0 & 23.50 & 400.0 & 0.10 & 70 & 1.0 & $10^{-7}$ \\
\hline
\end{tabular}

Figure 9 (d). Results are presented for the $\mathbf{u}-p_{w}$ and for the $\mathbf{u}-\theta-p_{w}$ formulations (the $\mathbf{u}-p^{\prime}-p_{w}$ gave similar results to the latter).

Although the cone resistance shows some scatter, Figure 9(a), a stationary state may be identified after a penetration of 10 radii. At that stage net cone resistance is approximately $155 \mathrm{kPa}$ for the $\mathbf{u}-\theta-p_{w}$ formulation and $170 \mathrm{kPa}$ for the $\mathbf{u}-p_{w}$ formulation. In Sheng et al. a value of $150 \mathrm{kPa}$ was obtained although using a different numerical approach. The $\mathbf{u}-\theta-p_{w}$ formulation results in a smoother penetration curve. This smoother response is even clearer on the pore-pressure curve, Figure 9(b), whereas the amplitude of the pore pressure oscillations is very large for the $\mathbf{u}-p_{w}$ formulation.

Figure 10 presents the values of water pressure and volumetric deformation obtained for both formulations at $z=20 R$. The small volumetric deformations confirm that the analysis is performed in almost undrained conditions. The water pressure field exhibits large gradients close to the $u_{2}$ position. This explains the oscillatory nature of the numerical record but not why the oscillations have smaller amplitude for the formulation. Indeed the distribution of the water pressure field is similarly smooth in both cases at the overall domain scale. This is consistent with the fact that both formulations use the FPL stabilization method in the mass balance equation.

The overall smoothness hides some local differences. In the $\mathbf{u}-p_{w}$ formulation some oscillations appear just below the tip of the cone; in addition, along the shaft of the penetrometer, slightly higher water pressures are found compared to the other formulation. Also, in the $\mathbf{u}-\theta-p_{w}$ formulation, marginally higher volumetric deformations are obtained at the shaft, indicating slightly higher water pressure dissipation.

Local differences are more noticeable when the stress fields are considered. Figure 11 compares the stress state at the final penetration position for both formulations. Using the $\mathbf{u}-p_{w}$ formulation, the effective mean pressure, the deviatoric stress invariant and the preconsolidation stress, exhibit large oscillations near to the penetrometer; that is in areas where the soil has undergone large plastic shearing. On the other hand, when using the $\mathbf{u}-\theta-p_{w}$ formulation all these stress fields are smoother. This is consistent with the fact that the momentum equation is also stabilized for this formulation. Stress field oscillations close to the penetrometer affect also the normal contact stresses along the shaft: a smoother stress profile is obtained with the $\mathbf{u}-\theta-p_{w}$ formulation, see Figure 9. These local variations in normal contact stress are the likely cause behind the observed large amplitude oscillation of the pore pressure record at the $u_{2}$ position in the $\mathbf{u}-p_{w}$ formulation. The benefits of extended stabilization for this coupled case are therefore evident.

\section{Conclusions}

A numerical framework, based on PFEM, for the modelling of geomechanical problems at large strains has been presented. A poromechanical model where the governing equations are the linear momentum and mass balance of the mixture is employed. The constitutive model is based on well-established soil mechanics formulations (Cam-Clay with Houlsby hyperelasticity; Darcy's law). Additionally, to examine a classical formulation for undrained problems, a single-phase, elasto-plastic Tresca material description has been also studied.

These two material descriptions have in common a potential for incompressibility that may cause numerical problems if not addressed in the formulation of the problem. The focus of the paper has been the development of stabilized mixed formulations for low order finite elements.

Three different one-phase mixed formulations for the mechanical problem have been assessed: the $\mathbf{u}-p$, $\mathbf{u}-\theta-p$ and $\mathbf{u}-\theta$ formulations. In all of them Polynomial Pressure Projection (PPP) is applied to stabilize the scalar field variables. In the case examined (penetration of a rigid strip footing) all the alternatives 


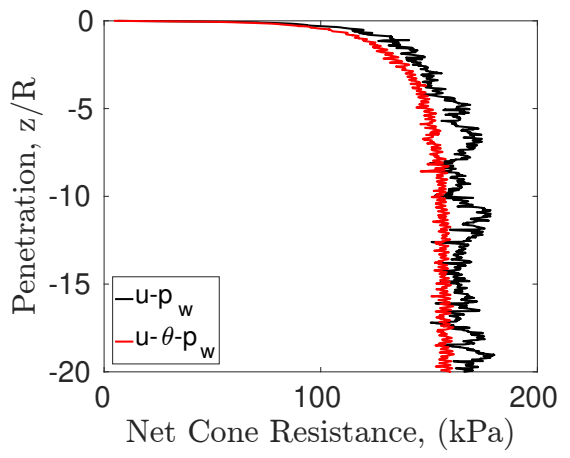

(a)

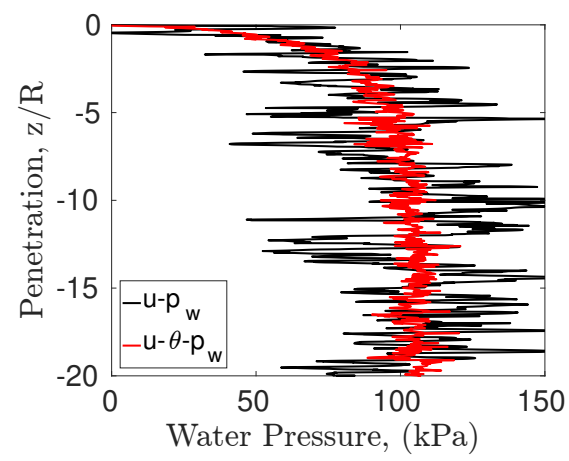

(b)

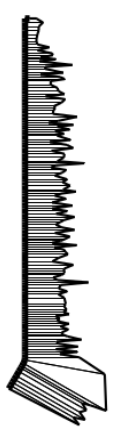

(c)

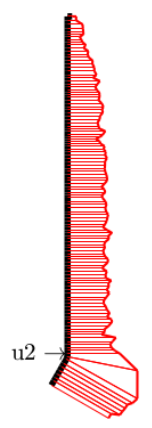

(d)

Figure 9: Cone Penetration Test. Evolution of the net cone resistance and water pressure at the $u_{2}$ position in terms of the dimensionless penetration depth, (a) and (b) respectively. Normal contact stress at the final penetration depth: $\mathbf{u}-p_{w}$ formulation, (c), and $\mathbf{u}-\theta-p_{w}$ formulation, (d).

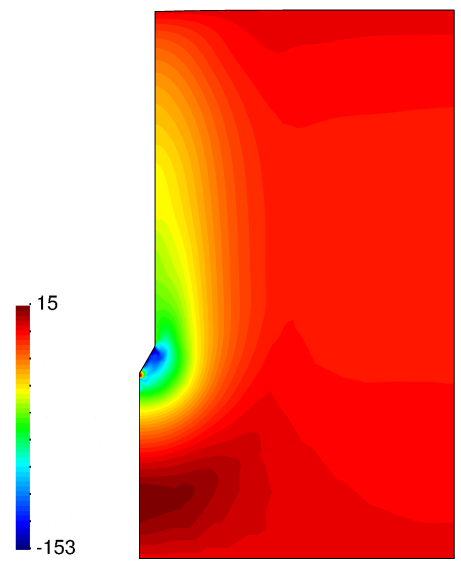

(a)

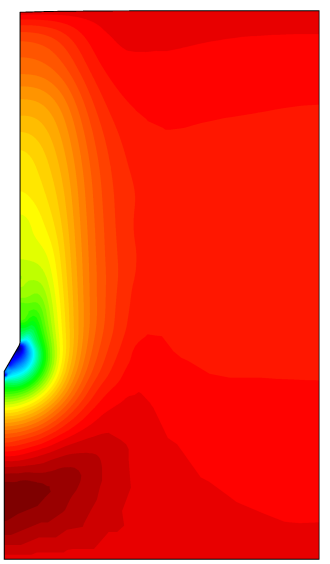

(b)

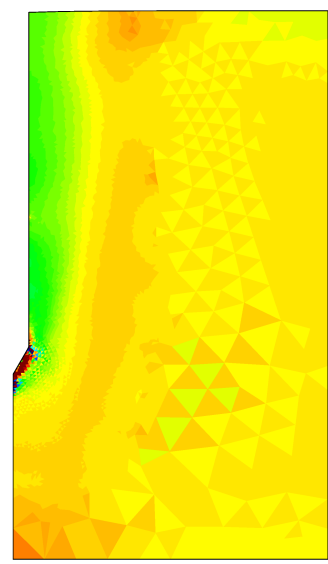

(c)

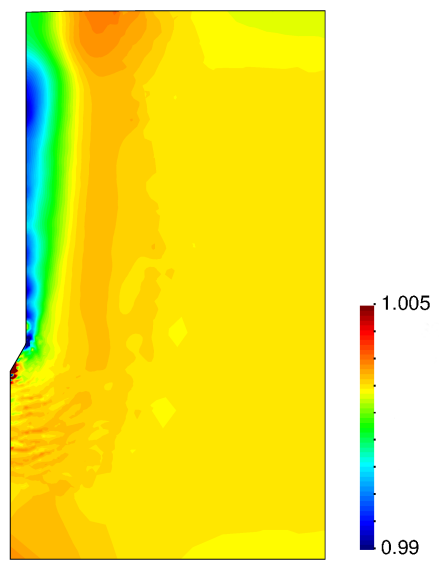

(d)

Figure 10: Cone Penetration Test. Contours for the water pressure (kPa), (a) and (b), the determinant of the deformation gradient at integration points, (c), and the nodal Jacobian ( $\theta$ ), (d). Results (a) and (c) correspond to the $\mathbf{u}-p_{w}$ formulation whereas results (b) and (d) correspond to the $\mathbf{u}-\theta-p_{w}$ formulation.

appear to perform well at the global response level. However, the formulation $\mathbf{u}-p$, which does not have the Jacobian as a nodal variable, presents significant spurious oscillations in the value of the Lode Angle. When the Jacobian is present no particular advantage is obtained from adding also the mean pressure $p$ as field variable.

Noting that the effective pressure and the specific volume change are here the relevant nodal variables, analogous mixed formulations - $\left(\mathbf{u}-p^{\prime}-p_{w}\right)$ and $\left(\mathbf{u}-\theta-p_{w}\right)$ - were explored for the poromechanical formulation. In addition to the PPP stabilization, a Fluid-Pressure-Laplacian stabilization of the mass conservation equation was also applied to the primal hydromechanical formulation, $\mathbf{u}-p_{w}$.

The performance of the alternative mixed formulations was similar as in the single-phase case. For the first example -a flexible circular footing resting on a Modified Cam Clay soil- a very similar global response was obtained in terms of water pressure and displacements. Significant differences between formulations arose in the more challenging final example, a CPTu in clay, that involves a contact problem with severe geometric non-linearities in almost undrained conditions. Using the primal formulation, with only a FPL 


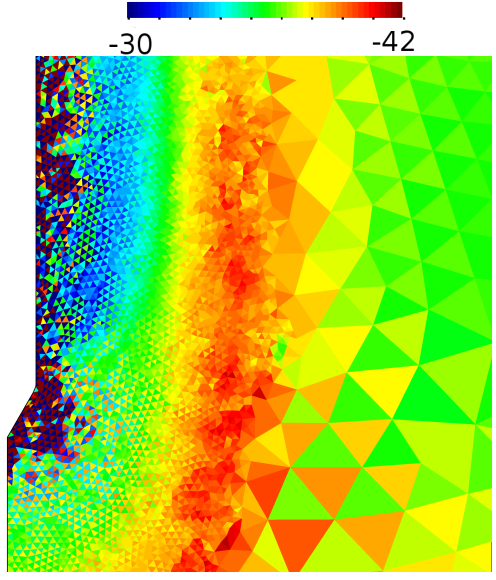

(a)

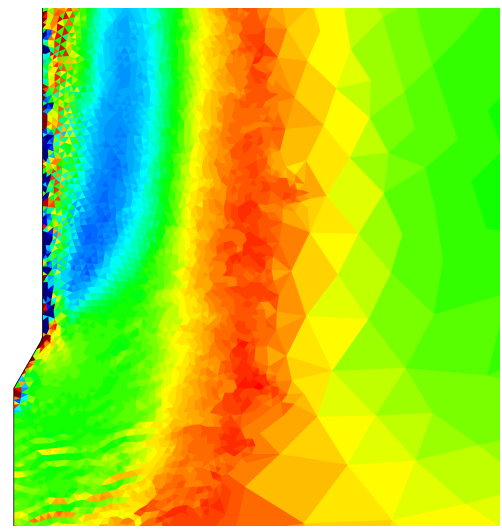

(d)

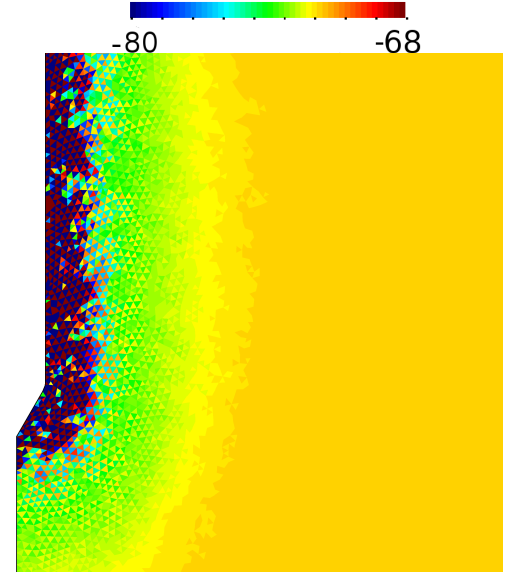

(b)

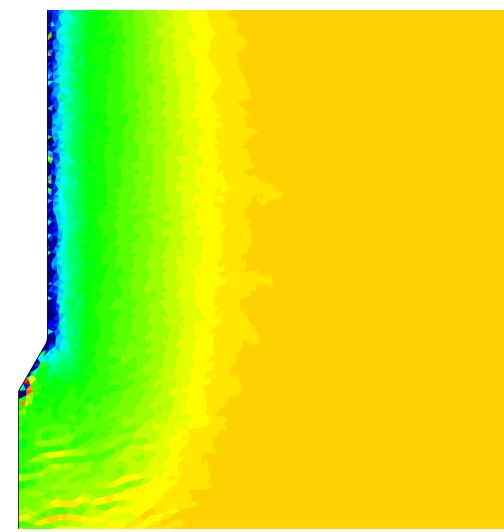

(e)

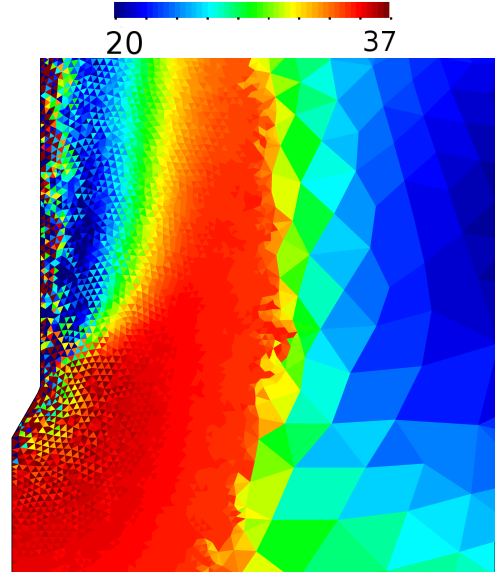

(c)

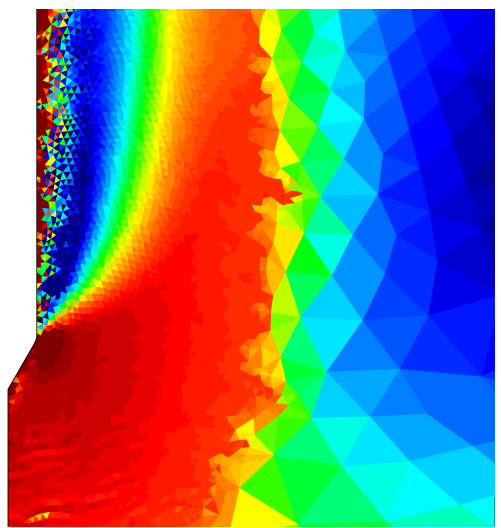

(f)

Figure 11: Cone Penetration Test. Contours, at integration points, of the effective pressure (kPa), (a) and (d), the preconsolidation pressure $(\mathrm{kPa}),(\mathrm{b})$ and $(\mathrm{e})$, and $J_{2}(\mathrm{kPa}),(\mathrm{c})$ and $(\mathrm{f})$. On top, the results obtained with the $\mathbf{u}-p_{w}$ formulation, and at the bottom, the results obtained with the $\mathbf{u}-\theta-p_{w}$ formulation.

mass-stabilization, was not enough to avoid high amplitude oscillations of the excess pore-pressure record at the -experimentally crucial- cone shoulder position. That was a consequence of oscillations in the stress fields in regions of large plastic deformations. The use of a Jacobian based mixed formulation $\left(\mathbf{u}-\theta-p_{w}\right)$ with extra PPP stabilization terms resulted in a significantly improved response. This better behaviour obtained with the $\mathbf{u}-\theta-p_{w}$ formulation makes this mixed form the best candidate to face soil mechanics problems within PFEM.

\section{Acknowledgements}

The support of the Ministry of Economy of Spain through research grants BIA2011-27217 and BIA201459467-R is gratefully acknowledged. 


\section{References}

[1] A. J. Abbo, A. V. Lyamin, S. W. Sloan and J. P. Hambleton. A C2 continuous approximation to the Mohr-Coulomb yield surface. International Journal of Solids and Structures, vol. 48(21), pp. 3001-3010 (2011).

[2] I. Babuška. Error-bounds for finite element method. Numerische Mathematik, vol. 16(4), pp. 322-333 (1971).

[3] K. J. Bathe. The inf-sup condition and its evaluation for mixed finite element methods. Computers $\mathcal{E}$ structures, vol. 79(2), pp. 243-252 (2010).

[4] P. B. Bochev, C. R. Dohrmann, and M. D. Gunzburger. Stabilization of Low-Order Mixed Finite Elements for the Stokes Equations. SIAM Journal on Numerical Analysis, vol. 44, pp. 82-101 (2008).

[5] R. I. Borja and E. Alarcón. A mathematical framework for finite strain elastoplastic consolidation Part 1: Balance laws, variational formulation, and linearization, Computer Methods in Applied Mechanics and Engineering, vol. 122(1-2), pp. 145-171 (1995).

[6] R. I. Borja and C. Tamagnini. Cam-Clay plasticity part III: Extension of the infinitesimal model to include finite strains. Computer Methods in Applied Mechanics and Engineering, vol 155(1-2), pp. 73-95 (1998).

[7] R. I. Borja, C. Tamagnini and E. Alarcón. Elastoplastic consolidation at finite strain part 2: finite element implementation and numerical examples, Computer Methods in Applied Mechanics and Engineering, vol. 159(1-2), pp. 103-122 (1998).

[8] F. Brezzi. On the existence, uniqueness and approximation of saddle-point problems arising from Lagrangian multipliers. Revue française d'automatique, informatique, recherche opérationnelle. Analyse numérique, vol. 8(2), pp. 129-151 (1974).

[9] J.M. Carbonell. Modeling of Ground Excavation with the Particle Finite Element Method. PhD thesis, Universitat Politècnica de Catalunya (UPC), Barcelona (2009).

[10] J. M. Carbonell, E. Oñate and B. Suárez. Modeling of Ground Excavation with the Particle FiniteElement Method. J. Eng. Mech, vol. 136(4), pp. 455-463 (2010)

[11] C. R. Dohrmann and P. B. Bochev. A stabilized finite element method for the Stokes problem based on polynomial pressure projections. International Journal for Numerical Methods in Fluids, vol. 46, pp. 183-201 (2004).

[12] H. Edelsbrunner and E.P. Mucke. Three dimensional alpha shapes. ACM Transaction on Graphics, vol. 13, pp. 43-72 (1994).

[13] G. T. Houlsby. The use of variable shear modulus in elasti-plastic models for clays. Computers and Geotechnics, vol 1(1), pp. 3-13 (1985).

[14] M. Kardani, M. Nazem, J. P. Carter and A. J. Abbo. Efficiency of High-Order Elements in LargeDeformation Problems of Geomechanics. Int. J. Geomech, vol. 15(6), pp. 04014101 (2015).

[15] A. Larese, R. Rossi, E. Oñate and S. R. Idelsohn. A coupled PFEM-Eulerian approach for the solution of porous FSI problems. Computational Mechanics, vol. 50(6), pp. 805-819 (2012)

[16] J. Larsson and R. Larsson. Non-linear analysis of nearly saturated porous media: theorical and numerical formulation. Comput. Methods Appl. Mech. Engrg., vol 191, pp 3885-3907 (2002). 
[17] L. Monforte, M. Arroyo, J. M. Carbonell and A. Gens. Numerical simulation of undrained insertion problems in Geotechnical Engineering with the Particle Finite Element Method (PFEM). Computers and Geotechnics, vol 82, pp 144-156 (2017).

[18] L. Monforte, M. Arroyo, A. Gens and J. M. Carbonell. Explicit finite deformation stress integration of the elasto-plastic constitutive equations. Computer Methods and Recent Advances in Geomechanics Proceedings of the 14th Int. Conf. of IACMAG, pp 267-272 (2014).

[19] J. Oliver, J. C. Cante, R. Weyler, C. González and H. Hernandez. Particle Finite Element Methods in Solid Mechanics Problems In: E. Oñate and R. Owen (Eds), Computational Plasticity, vol. 7. Springer, Netherlands, pp-87-103.

[20] E. Oñate, and S. R. Idelsohn, F. Del Pin and R. Aubry. The particle finite element method - an overview. International Journal of Computational Methods, vol. 1(2), pp- 267-307 (2004).

[21] E. Oñate, J. Rojek, R. L. Taylor, and O. C. Zienkiewicz. Finite calculus formulation for incompressible solids using linear triangles and tetrahedra. International Journal for Numerical Methods in Engineering, vol. 59(11), pp. 1473-1500 (2004).

[22] M. Pastor, T. Li, X. Liu, O.C. Zienkiewicz and M. Quecedo. A fractional step algorithm allowing equal order of interpolation for coupled analysis of saturated soil problems. Mechanics of Cohesive-frictional Materials, vol. 5(7), pp. 511-534 (2000).

[23] M. Preisig and J. H. Prévost. Stabilization procedures in coupled poromechanics problems: A critical assessment. International Journal for Numerical and Analytical Methods in Geomechanics, vol. 35(11), (2011).

[24] D. M. Potts and L. Zdravković. Finite element analysis in geotechnical engineering. Thomas Teldford, vol. 1, London (1999).

[25] M. M. Rashid. Material state remapping in computational solid mechanics. Int. J. Numer. Meth. Engng., vol. 55, pp. 431-450 (2002).

[26] P. A. Raviart and J. M. Thomas. A mixed finite element method for 2-nd order elliptic problems. Springer Berlin Heidelberg, pp. 292-315 (1977).

[27] P. K. Robertson. Interpretation of cone penetration tests-a unified approach. Canadian Geotechnical Journal, vol 46(11), pp. 1337-1355 (2009).

[28] J. M. Rodriguez, J. M. Carbonell, J. C. Cante, and J. Oliver. The particle finite element method (PFEM) in thermo-mechanical problems. Int. J. Numer. Meth. Engng, doi: 10.1002/nme.5186 (2016).

[29] M Rouainia and D. Muir Wood. Computational aspects in finite strain plasticity analysis of geotechnical materials. Mechanics Research Communications, vol 33(2), pp. 123-133 (2006).

[30] F. Salazar, J. Irazábal, A. Larese and E. Oñate Numerical modelling of landslide-generated waves with the particle finite element method (PFEM) and a non-Newtonian flow model. Int. J. Numer. Anal. Meht. Geomech., vol 40, pp. 809-836 (2016)

[31] D. Sheng, R. Kelly, J. Pineda and B. Lachlan. Numerical study of rate effects in cone penetration test. 3rd International Symposium on Cone Penetration Testing, pp. 419-428 (2014).

[32] J. C. Simo. Numerical analysis and simulation of plasticity. In: P. Ciarlet and J. Lions (Eds.), Handbook of Numerical Analysis, vol VI, pp. 183-499 (1998).

[33] J. C. Simo and T. J. R. Hughes Computational Inelasticity, Springer-Verlag, New York (1998). 
[34] S. W. Sloan, A. J. Abbo and D. Sheng. Refined explicit integration of elastoplastic models with automatic error control. Engineering Computations, vol. 18(1-2), pp. 121-194 (2001).

[35] E. A. de Souza Neto, D. Perić, M. Dutko, and D. R. J. Owen. Design of simple low order finite elements for large strain analysis of nearly incompressible solids. International Journal of Solids and Structures, vol 33(20), pp. 3277-3296 (1996).

[36] W. C. Sun, J. T. Ostien and A. G. Salinger. A stabilized assumed deformation gradient finite element formulation for strongly coupled poromechanical stimulations at finite strains. Int. J. Numer. Anal. Meth. Geomech., vol. 37, pp. 2755-2788 (2013).

[37] W. C. Sun, Q Chen and J. T. Ostien. Modeling the hydro-mechanical responses of strip and circular punch loadings on water-saturated collapsible geomaterials. Acta Geotechnica, vol 9(5), pp. 903-934 (2013).

[38] C. Taylor and P. Hood. A numerical solution of the Navier-Stokes equations using the finite element technique. Computers $\&$ Fluids, vol. 1(1), pp. 73-100 (1973).

[39] A. Truty and T. Zimmermann. Stabilized mixed finite element formulations for materially nonlinear partially saturated two-phase media. Computer Methods in Applied Mechanics and Engineering, vol. 195(13), pp. 1517-1546 (2006).

[40] X. Zhang, K. Krabbenhoft, D. Sheng, W. Li. Numerical simulation of a flow-like landslide using the particle finite element method. Computational Mechanics, vol. 55(1), pp. 167-177 (2015).

[41] O.C. Zienkiewicz and R. L. Taylor. The finite element method: solid mechanics. Butterworth-heinemann, vol. 2, Oxford (2000). 